ACCEPTED MANUSCRIPT

\title{
Impact of energetic particle orbits on long range frequency chirping of BGK modes
}

To cite this article before publication: Hooman Hezaveh Hesar Maskan et al 2017 Nucl. Fusion in press https://doi.org/10.1088/1741-4326/aa80a9

\section{Manuscript version: Accepted Manuscript}

Accepted Manuscript is "the version of the article accepted for publication including all changes made as a result of the peer review process, and which may also include the addition to the article by IOP Publishing of a header, an article ID, a cover sheet and/or an 'Accepted

Manuscript' watermark, but excluding any other editing, typesetting or other changes made by IOP Publishing and/or its licensors"

This Accepted Manuscript is @ 2017 IAEA, Vienna.

During the embargo period (the 12 month period from the publication of the Version of Record of this article), the Accepted Manuscript is fully protected by copyright and cannot be reused or reposted elsewhere.

As the Version of Record of this article is going to be / has been published on a subscription basis, this Accepted Manuscript is available for reuse under a CC BY-NC-ND 3.0 licence after the 12 month embargo period.

After the embargo period, everyone is permitted to use copy and redistribute this article for non-commercial purposes only, provided that they adhere to all the terms of the licence https://creativecommons.org/licences/by-nc-nd/3.0

Although reasonable endeavours have been taken to obtain all necessary permissions from third parties to include their copyrighted content within this article, their full citation and copyright line may not be present in this Accepted Manuscript version. Before using any content from this article, please refer to the Version of Record on IOPscience once published for full citation and copyright details, as permissions will likely be required. All third party content is fully copyright protected, unless specifically stated otherwise in the figure caption in the Version of Record.

View the article online for updates and enhancements. 


\title{
Impact of energetic particle orbits on long range frequency chirping of BGK modes
}

\author{
H. Hezaveh, Z. S. Qu, B. Layden and M. J. Hole \\ Research School of Physics and Engineering, The Australian National University, Canberra ACT
} 2601, Australia

E-mail: hooman.hezaveh@anu.edu.au

Abstract.

Long range frequency chirping of Bernstein-Greene-Kruskal modes, whose existence is determined by the fast particles, is investigated in cases where these particles do not move freely and their motion is bounded to restricted orbits. A nonuniform equilibrium magnetic field is included into the bumpon-tail instability problem of a plasma wave. The parallel field gradients account for the existence of different orbit topologies of energetic particles. With respect to fast particles dynamics, the extended model captures the range of particles motion (trapped/passing) with energy and thus represents a more realistic 1D picture of the long range sweeping events observed for weakly damped modes, e.g. global Alfven eigenmodes, in tokamaks. The Poisson equation is solved numerically along with bounce averaging the Vlasov equation in the adiabatic regime. We demonstrate that the shape and the saturation amplitude of the nonlinear mode structure depends not only on the amount of deviation from the initial eigenfrequency but also on the initial energy of the resonant electrons in the equilibrium magnetic field. Similarly, the results reveal that the resonant electrons following different equilibrium orbits in the nonuniform field lead to different rates of frequency evolution. As compared to the previous model [Breizman B.N. 2010 Nucl. Fusion 50 084014], it is shown that the frequency sweeps with lower rates. The additional physics included in the model enables a more complete 1D description of the range of phenomena observed in experiments. 


\section{Introduction}

Fast particles are abundantly present in burning plasmas. They exist either through external heating or eventually by fusion-born alpha particles. Energetic particle driven modes (EPMs) [1] can occur as a result of fast particles interaction with weakly damped plasma modes, e.g. Alfven eigenmodes (AEs) [2]. The resulted excited modes can cause the undesirable ejection of energetic particles from the hot core towards the walls of a toroidal machine $[3,4,5]$. This loss deteriorates plasma heating and degrades the confinement in a power plant. Accordingly, understanding the behavior of these modes is momentous in burning plasmas of future fusion reactors.

Experimental results, in the case of neutral beam injection, demonstrate that EPMs, as a result of AEs excitation, exhibit a hard nonlinear regime $[6,7,8,9$, 10] with rapid frequency sweeping. Small deviations from the initial eigenfrequency for the case of a nearthreshold instability $\left|\gamma_{l}-\gamma_{d}\right| \ll \gamma_{d} \leq \gamma_{l}$, where $\gamma_{l}$ is the kinetic drive and $\gamma_{d}$ is the damping rate due to dissipation in the background plasma, were first studied using a 1D bump-on-tail (BOT) model by Berk-Breizman (BB) and co-workers [11]. This model shows the nonlinear process of holes and clumps formation in the fast particle distribution function. A pair of Bernstein-Greene-Kruskal (BGK) [12] nonlinear modes chirping up and down in frequency is supported by these nonlinear phase-space structures and the frequency shifts are associated with the motion of these coherent structures due to energy dissipation in the bulk plasma. The much longer evolution time scale of these nonlinear structures in comparison with their development time scale in the explosive formation stage is one of the key results in [11] to be taken into consideration. It should be mentioned that holes and clumps form not only in case of a weakly unstable mode but also with any amount of background dissipation [13]. The Berk-Breizman scenario has been proved to be successful in explaining the frequency chirping events observed in experiments with AEs $[14,15]$. Moreover, the effect of different types of relaxation processes on the nonlinear evolution has been investigated in [16] and [17], with the BOT code introduced in the latter. All the mentioned models are based on the assumption that the range of frequency chirping is short and the mode structure is fixed.

However, experimental evidence exists for mode activities in which the frequency shifts are as large as the initial eigenfrequency itself $[18,19,20]$. As the mode amplitude saturates due to flattening of the distribution function of the energetic particles, the physical picture of each evolving phase-space structure is a BGK mode whose frequency changes in time and its structure is notably affected by the frequency shift. Recently, a nonperturbative model based on the adiabatic description of the fast particles contribution has been developed by Breizman [21] using a 1D BOT instability to interpret the long range chirping for an isolated nonlinear resonance. This approach is premised on the assumption that the width of the separatrix supported by the BGK mode is small compared with the characteristic width of the unperturbed distribution function. The Breizman model remains valid as long as the separatrix of the energetic particles inside the clump shrinks for a downward shift in the frequency. As an extension, the adiabatic description of treating an expanding separatrix which traps the ambient particles is presented in [22] by Nyquist and Breizman.

In magnetized plasmas, e.g. magnetic confinement devices, the particles gyrate about the magnetic field lines and follow certain trajectories depending on their energy and the magnetic field inhomogeneity. Therefore, the impact of particle orbits on the long range frequency sweeping events, should also be inyestigated in order to better understand and control these instability-driven phenomena. A physical system where the energetic particles are not moving freely and their equilibrium motion is bounded to certain orbits, enables such an investigation through a $1 \mathrm{D}$ picture. This physical model is the subject of this paper. We add a fixed nonuniform equilibrium magnetic field to the BOT problem presented in [21], thus creating an energy-dependence of the particle oscillation frequency through the mirror effect of the parallel field gradients. In this new model, the equilibrium field is pointed mainly in the z-direction and has $B_{\theta}=0$. For a $1 \mathrm{D}$ representation, we consider only the axis $(\mathrm{r}=0)$ of this magnetic mirror system and represent the magnetic field by

$$
B=B_{c}-B_{0} \cos \left(k_{\mathrm{eq}} z\right),
$$

where $k_{\text {eq }}$ is the spatial frequency of the magnetic field. We assume that the energetic particle confinement is due to the confinement of a single magnetic moment $(\mu)$ and treat the chirping of an unstable mode which has a low eigenfrequency compared to the ion cyclotron frequency and its wavelength is large compared to the electron Larmor radius of the resonant electrons. Therefore, the constants $B_{0}$ and $B_{c}$ are determined by

$$
B_{c}-B_{0} \gg\left[\frac{m_{i} \omega_{\mathrm{pe}}}{e Z_{i}}, \frac{m_{e} v_{\perp}}{e \lambda_{p}}\right]
$$

where $m_{i}$ and $m_{e}$ are the ion and electron mass, respectively, $\omega_{\text {pe }}$ is the electron plasma frequency, $Z_{i}$ is the number of ion charges, $e$ is the elementary charge, $v_{\perp}$ is the velocity of fast particle perpendicular to the magnetic field and $\lambda_{p}$ is the wavelength of the perturbed mode. In this new model, the unperturbed 
guiding center motion of the fast particles in the equilibrium field is governed by the following orbitaveraged Littlejohn's Hamiltonian [23]:

$$
H_{0}=\frac{p_{z}^{2}}{2 m_{e}}-\mu B_{0} \cos \left(k_{\mathrm{eq}} z\right)+\mu B_{c}
$$

where $p_{z}$ is the energetic particles momentum in the $z$-direction and it is assumed $A_{z}=0$ with $A$ the vector potential. The energetic particles interacting with the perturbed field are considered as trapped or passing in this magnetic mirror system, depending on their pitch angle. Figure 1, whose construction is detailed at the end of Subsection 2.1, demonstrates the behavior of the equilibrium oscillation frequency of the fast electrons versus their energy. For each frequency of trapped particles motion in the equilibrium magnetic field, there exist a group of passing particles having the same frequency of the equilibrium motion. Hence, the mode can be simultaneously in resonance with both the trapped and passing electrons in this equilibrium field. This trapped and passing locus model resembles the trapped particles following the banana orbits and the passing particles in the magnetic field lines of a tokamak (Cf. Section 5). In addition to enabling the impact of particle orbits on the long range chirping of BGK modes, the contribution from different resonances can also be investigated through the energy dependence.

The nonlinear wave equation is expanded using Fourier decomposition which allows us to find an explicit expression for the Hamiltonian of the fast particles motion in terms of the action-angle variables of the unperturbed motion. This expansion, together with treating the kinetic equation adiabatically, allows us to implement a numerical treatment to investigate the impact of particle orbits on the structure and the sweeping rate of the nonlinear wave

In Section 2, the basic system of equations adopted for the analysis and the dynamic equations of the unperturbed motion is presented, followed by the derivation of the linear growth rate, the equation for the BGK mode structure and the chirping rate. The numerical scheme used for solving the equations is assigned to Section 3. Section 4 presents the results in the regions where the adiabatic invariant of the trapped particles in the BGK mode decreases (the separatrix shrinks) during chirping and the effect of the electrons equilibrium orbit on the nonlinear evolution of the mode. Finally, Section 5 contains concluding remarks.

\section{The model}

In this extended 1D BOT model, we study a purely electrostatic mode in a plasma consisting of static

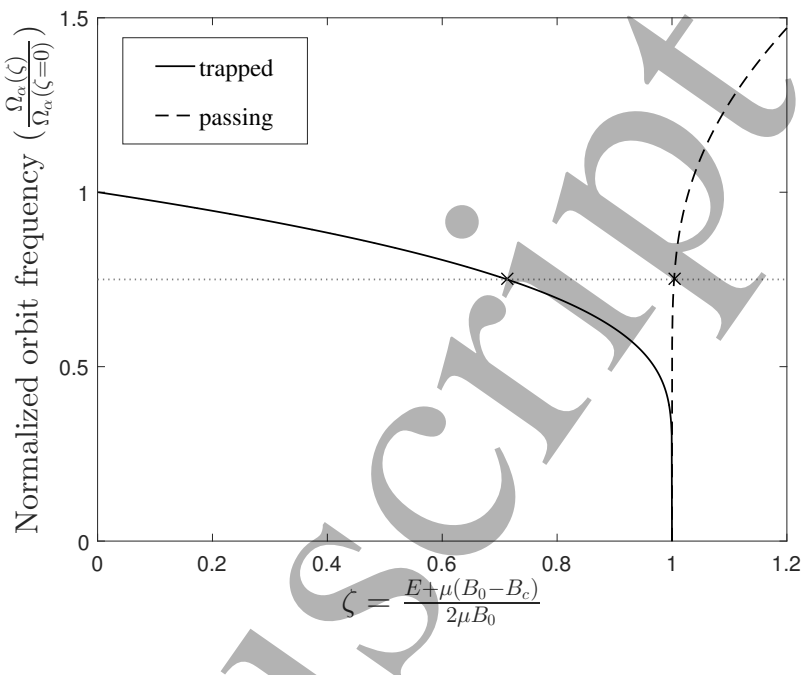

Figure 1. Normalized equilibrium frequency of the fast particles in the nonuniform magnetic field vs. energy parameter. The dotted line shows a sample eigenfrequency simultaneously in resonance with particles of two different orbit types

background ions, cold electrons responding linearly to the mode and fast electrons which are trapped and co/counter-passing in the nonuniform magnetic field and are in resonance with the electrostatic mode. We focus on propagation and dynamics parallel to the equilibrium magnetic field where the cold plasma has an isotropic distribution and its density will be uniform along the magnetic field. The distribution function of each group of the fast electrons, which is treated through the Vlasov equation, is assumed to be a linearly increasing function of the fast electrons energy providing the instability drive. The damping mechanism is modelled by a Krook collision model, which induces frequency chirping behavior when affecting the resonant energetic electrons as an energy sink. The system of equations considered to investigate both the linear evolution of the mode and the structure of the BGK mode during frequency chirping consists of Poisson, Vlasov, equation of motion and continuity equation, given by

$$
\begin{aligned}
& \frac{\epsilon_{0}}{e} \frac{\partial^{2} U}{\partial z^{2}}=-e\left[\sum_{\alpha} \int \tilde{f}_{\alpha} d v+\delta n_{c}\right], \\
& \frac{\partial f_{\alpha}}{\partial t}+\left\{f_{\alpha}, H_{\alpha}\right\}=0, \\
& \frac{\partial V_{c}}{\partial t}=-\frac{1}{m_{e}} \frac{\partial U}{\partial z}-\nu V_{c}, \\
& \frac{\partial \delta n_{c}}{\partial t}=-n_{c} \frac{\partial V_{c}}{\partial z}
\end{aligned}
$$

with $\alpha$ a label that denotes the orbit type of the fast electrons motion in the magnetic field: $(\alpha=\mathbf{T})$ and $(\alpha=\mathbf{P})$ for the trapped and passing electrons in this field, respectively. The Poisson bracket is denoted by \{\} in equation (4b). The total distribution function of 
energetic electrons is $f_{\alpha}=F_{\text {eq }, \alpha}+\tilde{f}_{\alpha}$, with $F_{\text {eq }, \alpha}$ and $\tilde{f}_{\alpha}$ being the initial and the perturbed parts, respectively. The energy of the electrostatic mode is given by $U$, $\epsilon_{0}$ is the permittivity of free space, $\nu=2 \gamma_{d}$ is the Krook collision frequency of the cold electrons, $V_{c}$ is the flow velocity of the cold electrons and $n_{c}$ and $\delta n_{c}$ are the unperturbed and perturbed density of the cold electrons, respectively.

\subsection{Fast particles orbits and dynamics}

For the completely integrable system consisting of trapped and co/counter-passing particles whose motion is goverened by the Hamiltonian presented in equation (3), it is possible to transform canonically from the variables $\left(z, p_{z}\right)$ to action-angle variables $\left(\theta, J_{\alpha}\right)$, written as

$$
\begin{aligned}
& J_{\alpha=\mathbf{T}}=\frac{1}{2 \pi} \oint p_{z} d z \\
& =\frac{2}{\pi} \int_{0}^{z \max } \sqrt{2 m_{e}\left[E-\mu B_{c}+\mu B_{0} \cos \left(k_{\mathrm{eq}} z\right)\right]} d z \\
& =\frac{8 \sqrt{m_{e} \mu B_{0}}}{k_{\mathrm{eq}} \pi}[(\zeta-1) \mathbb{K}(\zeta)+\mathbb{E}(\zeta)] \\
& J_{\alpha=\mathbf{P}}=\frac{1}{2 \pi} \int_{0}^{\lambda} p_{z} d z=\frac{4 \sqrt{\mu B_{0}}}{k_{\mathrm{eq}} \pi} \mathbb{E}\left(\zeta^{-1}\right),
\end{aligned}
$$

where $J_{\alpha}$ is the action for the unperturbed motion of the fast particles, $z_{\max }$ is determined by $p_{z}=0$ using equation (3), $\lambda$ is the wavelength of the equilibrium field, $E$ is the unperturbed energy denoting the orbits, $\zeta$ is the energy parameter given by

$$
\zeta=\frac{E+\mu\left(B_{0}-B_{c}\right)}{2 \mu B_{0}}
$$

and $\mathbb{K}(\zeta)$ and $\mathbb{E}(\zeta)$ are the complete elliptic integral of the first and second kind, respectively, given by

$$
\begin{array}{r}
\mathbb{K}(\zeta)=\int_{0}^{\frac{\pi}{2}} \frac{d \varrho}{\sqrt{1-\zeta \sin ^{2} \varrho}} \\
\mathbb{E}(\zeta)=\int_{0}^{\frac{\pi}{2}} \sqrt{1-\zeta \sin ^{2} \varrho d \varrho .}
\end{array}
$$

Using the canonical equations of motion, the frequency of the motion reads

$$
\begin{aligned}
& \Omega_{\alpha=\mathbf{T}}=\frac{\partial H_{0, \alpha=\mathbf{T}}}{\partial J_{\alpha=\mathbf{T}}}=\frac{k_{e q} \pi \mu B_{0}}{2 \mathbb{K}(\zeta) \sqrt{m_{e} \mu B_{0}}}, \\
& \Omega_{\alpha=\mathbf{P}}=\frac{k_{e q} \pi \mu B_{0} \sqrt{\zeta}}{\mathbb{K}\left(\zeta^{-}-1\right) \sqrt{m_{e} \mu B_{0}}},
\end{aligned}
$$

The behavior of these frequencies (shown in figure 1) is similar to the bounce or transit frequency of the guiding center motion in tokamaks [24].

\subsection{The linear growth rate}

In this subsection, we investigate the linear interaction between the plasma mode and the fast particles that are trapped and co/counter-passing in the equilibrium magnetic field. For a traveling wave solution, the general form of the physical quantities can be represented as $U=\sum_{n=1}^{\infty} \frac{e \phi_{n}}{2} \exp \left[i n\left(k_{p} z-\omega t\right)\right]+c \cdot c=$ $\sum_{n=1}^{\infty} \frac{e \phi_{n}}{2} \sum_{p=-\infty}^{\infty} V_{\alpha, n, p}\left(J_{\alpha}\right) \exp [i(p \theta-n \omega t)]+c . c$ and $\tilde{f}_{\alpha}=\sum_{n=1}^{\infty} \sum_{p=-\infty}^{\infty} \hat{f}_{\alpha, n, p}\left(J_{\alpha}\right) \exp [i(p \theta-n \omega t)]+$ c.c, where $\omega=\omega_{r}+i \gamma_{l}$ is the complex frequency, $k_{p}$ the wave-number of the plasma mode, $V_{\alpha, n, p}\left(J_{\alpha}\right)$ the orbit averaged mode amplitude which specifies the coupling strength and plays the same role as the so-called matrix element in $[25,26]$, given by

$$
\begin{aligned}
V_{\alpha, n, p} & =\frac{1}{2 \pi} \int_{-\pi}^{\pi} \exp \left(i n k_{p} z\right) \exp (-i p \theta) d \theta \\
& =\frac{1}{2 \pi} \int_{-\pi}^{\pi} \cos \left(n k_{p} z-p \theta\right) d \theta,
\end{aligned}
$$

where $z(J, \theta)$ is presented in Appendix A and we have used the property that $z$ is an odd function of $\theta$ (see figure 5). In the previous BOT models for long range chirping $[21,27,22], V_{\alpha, n, p}$ is unity for the dominant resonance and is zero otherwise. In contrast, the presented approach enables investigation of different types of resonances in wave-particle interaction through a 1D model. It is noteworthy that the value of $k_{p} / k_{\text {eq }}=m$, where $m$ is an integer, can be associated with the mode numbers in realistic geometries.

The total Hamiltonian describing the fast particle motion can be written in the form, $H_{\alpha}=H_{0, \alpha}+$ $U$. This Hamiltonian along with the linearization of equation (4b), is used to derive the linearized Vlasov equation in the form given by

$$
\frac{\partial \tilde{f}_{\alpha}}{\partial t}+\frac{\partial \tilde{f}_{\alpha}}{\partial \theta} \frac{\partial H_{0, \alpha}}{\partial J_{\alpha}}=\frac{\partial F_{\mathrm{eq}, \alpha}\left(J_{\alpha}\right)}{\partial J_{\alpha}} \frac{\partial U}{\partial \theta} .
$$

Neglecting the higher harmonics $(n \geq 2)$ in the linear approximation,

$$
\hat{f}_{\alpha, n=1, p}=\frac{p e \phi_{n=1} V_{\alpha, n=1, p}\left(J_{\alpha}\right) \frac{\partial F_{\mathrm{eq}}\left(J_{\alpha}\right)}{\partial J_{\alpha}}}{2\left(p \Omega_{\alpha}-\omega\right)} .
$$

It can be infered from expression (11) that the resonance condition is

$$
\omega_{r}=p \Omega_{\alpha} .
$$

The sign of $\Omega_{\alpha}$ is affected by the definition of the angle and considering $\Omega_{\alpha}>0$, the resonance condition will be satisfied only for $p>0$. This means the lower summation index for $p$ in $U$ and $\tilde{f}_{\alpha}$ should be set to 
one and the negative values of $p$ correspond to nonresonant particles.

The perturbed density of the cold electrons can be derived from the linear fluid equations, (4c) and (4d). To first order in perturbations, we have

$$
\begin{array}{r}
V_{c}=\frac{k_{p} U}{\omega m_{e}}, \\
\delta n_{c}=\frac{k_{p}^{2} n_{c} U}{m_{e} \omega^{2}} .
\end{array}
$$

Now we substitute the relevant terms into (4a) to find the dispersion relation of the mode given by

$$
\begin{aligned}
\frac{\epsilon_{0} k_{p} m_{e}}{e^{2}} & \left(1-\frac{\omega_{\mathrm{pe}}^{2}}{\omega^{2}}\right)= \\
& \sum_{\alpha} \int \sum_{p} \frac{p\left(\frac{\partial F_{\mathrm{eq}, \alpha}}{\partial J_{\alpha}}\right)}{p \Omega_{\alpha}-\omega} V_{\alpha, n=1, p}^{2} d J_{\alpha},
\end{aligned}
$$

where $\omega_{\mathrm{pe}}=\sqrt{\frac{n_{c} e^{2}}{m_{e} \epsilon_{0}}}$ is the electron plasma frequency. Neglecting the small contribution of the principal value which does not modify the real part of the frequency significantly, allows us to set $\omega_{r}=\omega_{\text {pe }}$. Assuming $\gamma_{l} \ll \omega_{\mathrm{pe}}$ (the wave evolves slowly compared with $\omega_{\mathrm{pe}}^{-1}$ ), equation (14) can be solved for $\omega$. Consequently, the linear growth rate is found to be

$$
\begin{aligned}
\gamma_{l}= & \frac{\omega_{\mathrm{pe}} \pi e^{2}}{2 \epsilon_{0} k_{p} m_{e}} \sum_{\alpha} \sum_{p}\left[\frac{\partial F_{\mathrm{eq}, \alpha}}{\partial \zeta_{\alpha}} V_{\alpha, n=1, p}^{2}\right. \\
& \left.\times\left|\frac{\mathrm{d} \Omega_{\alpha}}{\mathrm{d} \zeta_{\alpha}}\right|_{\Omega_{\alpha}\left(J_{\alpha}\right)=\frac{\omega \mathrm{pe}}{p}}^{-1}\right]
\end{aligned}
$$

which involves summing the contribution from all the resonances denoted by p. Equation (15) is a function of the energy parameter $(\zeta)$. This indicates the dependency of the linear growth rate on particle orbits (see figure 3). It should be noted that the contribution from the counter-passing electrons in the equilibrium field is much less than the co-passing ones. This can be shown by changing $z$ to $-z$ in equation (9) and evaluating the corresponding values of the coupling strength for counter-passing eleetrons numerically.

\subsection{Nonlinear BGK modes}

Nonlinear frequency chirping can occur in unstable systems both near or far from marginal stability, in the absence of collisions. For a near-threshold instability, the presence of dissipation leads to the formation of an unstable plateau in the distribution function of the energetic electrons which supports sideband oscillations that finally evolve into chirping modes $[11,13]$. In this case, the chirping mode emerges nearly immediately near the marginal stability. However, for the case of a far from threshold instability, the system is so unstable that many modes are likely to be excited. If modes are comparable in frequency with overlapping eigenfunctions, this may lead to mode overlap. Simple chirping can however naturally occur in experiment when the system first goes unstable where there is only a discrete number of unstable modes that can arise from a near continuum of damped modes. Accordingly, we consider the case of a nearthreshold instability. The condition $\frac{\mathrm{d} \omega}{\mathrm{d} t}<\omega_{b}^{2}$, with $\omega(t)$ the frequency of the BGK mode and $\omega_{b}$ the bounce frequency of trapped electrons in this mode, ensures the existence of a trapping structure with a hole/clump in the phase-space of energetic particles. After development, the time scale of the motion of already established holes and clumps is much longer than the time scale of the energetic particles motion trapped in the BGK mode, i.e. $\omega_{b}^{-1}[11,28]$. In the present model, we focus on the adiabatic description of nonlinear BGK modes and construct our formalism based on the limit $\left[\frac{\mathrm{d} \omega_{b}}{\mathrm{~d} t}, \frac{\mathrm{d} \omega}{\mathrm{d} t}\right] \ll \omega_{b}^{2} \sim \gamma_{l}^{2} \sim \gamma_{d}^{2}$, where the kinetic equation can be bounce-averaged to find the perturbed distribution function of the fast electrons. The adiabatic limit should, in general, be checked if it remains valid as the frequency deviates from the initial eigenfrequency $[28,29,30]$.

Adopting a Fourier expansion for the periodic structure, the electrostatic energy of the nonlinear BGK mode can be written in the form

$$
U[z, t]=\sum_{n} A_{n}(t) \cos \left[n\left(k_{p} z-\phi(t)\right)\right],
$$

where the Fourier coefficients $A_{n}(t)$ evolve on a slow time scale but the periodic behavior of the BGK mode represents rapid oscillations with a time scale on the order of the inverse initial plasma frequency. The motion of the fast electrons can be investigated using the following Hamiltonian

$$
\begin{aligned}
H_{\alpha} & =H_{0, \alpha}\left(J_{\alpha}\right)+\frac{1}{2} \sum_{n} \sum_{p} A_{n}(t) \\
& \times V_{\alpha, n, p} \exp [i(p \theta-n \phi(t))]+c . c,
\end{aligned}
$$

written in terms of the action-angle variables of the unperturbed motion. A simple canonical transformation can be used to cancel the fast time scale included in $\phi(t)$. We consider $\tilde{\theta}_{l}=l \theta-\phi(t)$ and $\tilde{J}_{\alpha}=$ $\frac{J_{\alpha}}{l}$ and the type 2 generating function for this canonical transformation is $\Phi\left[\theta, \tilde{J}_{\alpha}, t\right]=l \theta \tilde{J}_{\alpha}-\phi(t) \tilde{J}_{\alpha}$, where $l=\frac{p}{n}$ denotes the type of the resonance. Considering the first resonance as having the dominant contribution to the interaction, the model can be evaluated by setting $l=1$. In section 4 , it is discussed that the contribution from the first resonance is dominant in this model. However, other types of resonances can be 
treated likewise. The new Hamiltonian is

$$
\begin{aligned}
K_{\alpha}(\tilde{\theta}, \tilde{J}, t) & =H_{0, \alpha}\left(\tilde{J}_{\alpha}\right)-\frac{\mathrm{d} \phi(t)}{\mathrm{d} t} \tilde{J}_{\alpha}+ \\
& \frac{1}{2} \sum_{n} A_{n}(t) V_{\alpha, n, n} \exp (i n \tilde{\theta})+c . c .
\end{aligned}
$$

The small separatrix width assumption allows us to neglect the higher order terms in the Taylor expansion of the unperturbed Hamiltonian near the resonant orbit. In addition, we also approximate $V_{\alpha, n, n}(\tilde{J})$ with the first term of its Taylor expansion about the value of action variable at resonance, denoted by $J_{\text {res, } \alpha}$. Using $\left.\frac{\partial H_{0, \alpha}}{\partial \tilde{J}_{\alpha}}\right|_{\tilde{J}_{\alpha}=J_{\mathrm{res}, \alpha}(t)}=\Omega_{\alpha}=\frac{\mathrm{d} \phi(t)}{\mathrm{d} t}=\omega(t)$, the new Hamiltonian becomes

$$
\begin{aligned}
K_{\alpha}= & \left.\frac{1}{2} \frac{\partial^{2} H_{0, \alpha}}{\partial \tilde{J}_{\alpha}^{2}}\right|_{\tilde{J}_{\alpha}=J_{\mathrm{res}, \alpha}(t)}\left(\tilde{J}_{\alpha}-J_{\mathrm{res}, \alpha}(t)\right)^{2}+ \\
& \frac{1}{2} \sum_{n} A_{n}(t) V_{\alpha, n, n} \exp (i n \tilde{\theta})+c . c .
\end{aligned}
$$

Substituting $K_{\alpha}$ with the extremum value of the BGK mode electrostatic energy in equation (19), gives the dynamics of the fast electrons on the separatrix supported by the nonlinear mode. This condition
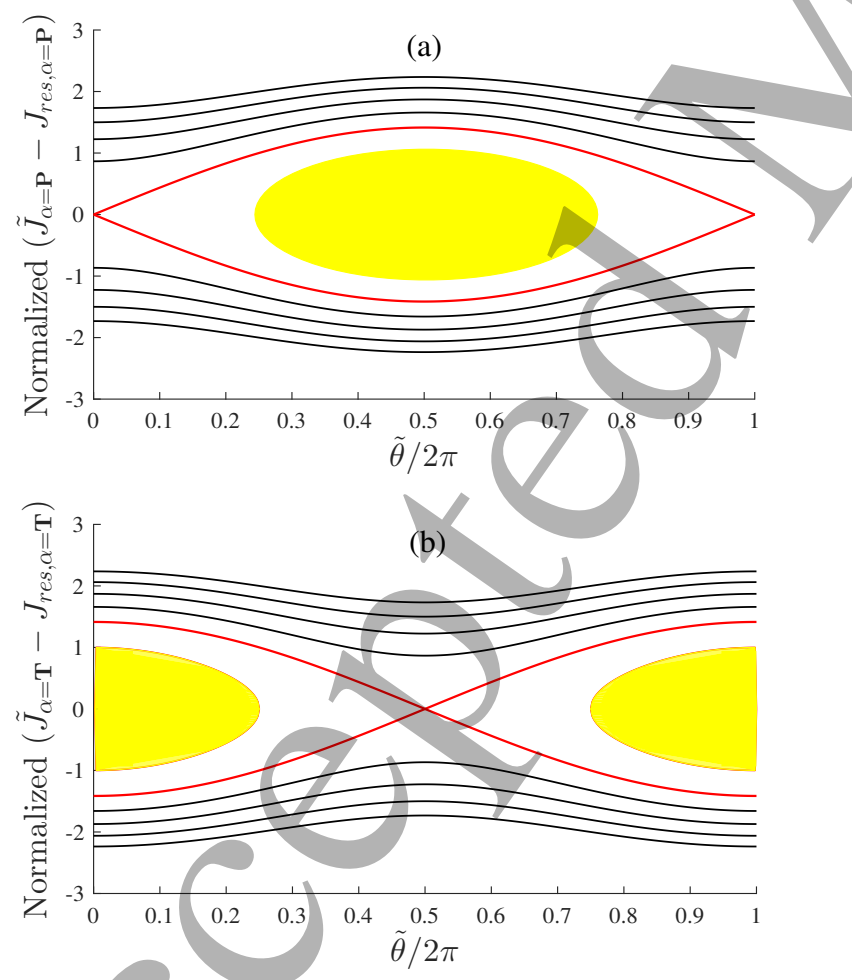

Figure 2. The panels (a) and (b) describe energy contours in phase space of the electrons which are passing and trapped in the equilibrium field, respectively. The black lines specifiy the trajectories of the passing electrons and the shaded area is a sample of the adiabatic invariant of the trapped electrons in the nonlinear BGK mode. The red line is the separatrix. is used to identify the boundary of the trapped and passing electrons in the BGK mode, i.e. the separatrix. A simple manipulation of equation (19) gives

$$
\begin{aligned}
\tilde{J}_{\alpha, \pm} & -J_{\mathrm{res}, \alpha}(t)= \\
\pm & {\left[\left(U_{\mathrm{ext}, \alpha}-\frac{1}{2} \sum_{n} A_{n}(t) V_{\alpha, n, n}\left(J_{\mathrm{res}, \alpha}\right)\right.\right.} \\
\times & \left.\exp (i n \tilde{\theta})+c . c) \frac{2}{\Delta_{\alpha}}\right]^{\frac{1}{2}}
\end{aligned}
$$

where $U_{\alpha, \text { ext }}$ is the extremum value of the BGK mode energy. The value of $\left.\frac{\partial^{2} H_{0, \alpha}}{\partial \tilde{J}_{\alpha}^{2}}\right|_{\tilde{J}_{\alpha}=J_{\text {res }, \alpha}(t)}=$ $\left.\frac{\partial \Omega_{\alpha}}{\partial \tilde{J}_{\alpha}}\right|_{\tilde{J}_{\alpha}=J_{\text {res }, \alpha}(t)}$ (denoted by $\Delta_{\alpha}$ ) can be negative or positive for the trapped or passing electrons in the equilibrium field, respectively. Mathematically, this affects $U_{\text {ext, } \alpha}$ in order to have a positive value under the square root in equation (20) and from the physical point of view, it shows that the passing electrons in the magnetic field/are trapped in the energy well of the BGK mode, while the trapped electrons in this field are trapped in the energy hill of the BGK mode. This implies

$$
U_{\text {ext }, \alpha}=\left\{\begin{array}{ll}
U_{\min }, & \alpha=\mathbf{T} \\
U_{\max }, & \alpha=\mathbf{P}
\end{array} .\right.
$$

Phase-space trajectories of constant energy for the motion of energetic particles in the BGK mode are plotted in figure 2. It is shown that the separatrix supported by the nonlinear mode corresponding to the electrons trapped in the equilibrium magnetic field (figure 2(b)) has a phase shift of $\pi$ with respect to the separatrix related to the passing group (figure 2(a)).

As the separatrix moves adiabatically, the phasespace area enclosed by the trajectories of the deeply trapped particles in the nonlinear wave, i.e. the shaded areas in figure 2, is conserved. Without trapping or detrapping over this region, the aforementioned conservation ensures that the value of the distribution function is conserved. The separatrix moves the trapped electrons in the BGK mode while the passing electrons are affected through the direction of their motion [21]. The adiabatic invariant of the motion of these electrons in the BGK mode reads (see Appendix B for more details)

$$
\begin{aligned}
I_{\alpha}= & 2 \int_{0}^{2 \pi}\left[\left(K_{\alpha}-\frac{1}{2} \sum_{n} A_{n}(t) V_{\alpha, n, n}\right.\right. \\
& \left.\times \exp (i p \tilde{\theta})+c . c) \frac{2}{\Delta_{\alpha}}\right]^{\frac{1}{2}} d \tilde{\theta} .
\end{aligned}
$$


Hard nonlinear evolution of BGK modes in the presence of fast particle orbits

Substituting expression (16) into equation (4a) gives

$$
\begin{array}{r}
-\sum_{n} A_{n}(t) n^{2} k_{p}^{2} \cos [n(k z-\phi(t))]=-\frac{e^{2}}{\epsilon_{0}} \\
\times\left[\frac{1}{m_{e}} \sum_{\alpha} \int_{-\infty}^{\infty} \tilde{f}_{\alpha}\left(z, p_{z}\right) d p_{z}+\delta n_{c}\right],
\end{array}
$$

where $\delta n_{c}$ can be derived under the linear response assumption of the bulk electrons. Similar to subsection 2.2 , we multiply equation $(23)$ by $\cos \left[n\left(k_{p} z-\phi(t)\right)\right]$ and integrate over one wave-length. We also write all the physical quantities in the fast particle term in terms of the new action-angle variables $(\tilde{\theta}, \tilde{J})$. After substituting the Fourier expansion of $\cos [n(k z(\tilde{\theta}, \tilde{J})-\phi(t))]$ and neglecting the highly oscillating terms one finds

$$
\begin{aligned}
& A_{n}(t)=\frac{1}{2 \pi k_{p} n_{c}}\left[\frac{\omega^{2}}{n^{2} \hat{\omega}^{2}-1}\right] \sum_{\alpha} \int_{0}^{2 \pi} d \tilde{\theta} \int_{0}^{\infty}\left[\tilde{f}_{\alpha}(\tilde{\theta}, J)\right. \\
& \left.\quad \times V_{\alpha, n, n} \exp (i n \tilde{\theta})+c . c\right]|\mathbf{J}| d \tilde{J},
\end{aligned}
$$

where the Jacobian of the canonical transformation $\left(z, p_{z}\right) \leftrightarrow(\tilde{\theta}, \tilde{J})$ is unity and $\hat{\omega}=\frac{\omega}{\omega_{\mathrm{pe}}}$ is the normalized frequency with respect to the initial electron plasma frequency. In this model, the phasespace density of the fast electrons (the distribution function) is assumed to be the same inside the narrow shrinking separatrix supported by the BGK mode, the so-called top-hat model. The perturbed part of the fast electrons distribution function dominated by the trapped electrons inside the separatrix [21] is calculated using the bounce averaging method described in Appendix B,

$\tilde{f}_{\alpha}=$ $\begin{cases}0, & \text { passing in BGK } \\ F_{\text {eq }, \alpha}\left(J_{\text {res }}(t=0)\right)-F_{\text {eq }, \alpha}\left(J_{\text {res }}(t)\right) . & \text { trapped in BGK }\end{cases}$

Using the above expression, equation (24) transforms into

$$
\begin{gathered}
A_{n}(t)=\frac{\omega^{2}}{2 \pi k n_{c}\left(n^{2} \hat{\omega}^{2}-1\right)} \sum_{\alpha}\left[F_{\mathrm{eq}, \alpha}(t=0)-F_{\mathrm{eq}, \alpha}(t)\right] \\
\times \int_{0}^{2 \pi} d \tilde{\theta}\left[V_{\alpha, n, n} \exp (i n \tilde{\theta})+c . c\right] \Delta \tilde{J}_{\alpha, \max }(\tilde{\theta}), \quad(26)
\end{gathered}
$$

where $\Delta \tilde{J}_{\alpha, \max }(\tilde{\theta})$ is the width of the separatrix. Using equation (20), we have

$$
\begin{gathered}
A_{n}(t)=\frac{\omega^{2}}{\pi k n_{c}\left(n^{2} \hat{\omega}^{2}-1\right)} \sum_{\alpha}\left[F_{\mathrm{eq}, \alpha}(t=0)-F_{\mathrm{eq}, \alpha}(t)\right] \\
\times \int_{0}^{2 \pi}\left[\left(U_{\alpha, \mathrm{ext}}-\frac{1}{2} \sum_{n} A_{n}(t) V_{\alpha, n, n} \exp (i n \tilde{\theta})\right.\right. \\
\left.+ \text { c.c }) \frac{2}{\Delta_{\alpha}}\right]^{\frac{1}{2}}\left[V_{\alpha, n, n} \exp (i n \tilde{\theta})+c . c\right] d \tilde{\theta} .
\end{gathered}
$$

The above equation can be solved numerically to derive the Fourier coefficients with which we can construct the structure of the plane wave. The numerical method used is presented in section 3 .

The trapped electrons in the BGK mode travel in phase-space together with the nonlinear mode. Depending on whether the clumps are trapped or passing in the equilibrium field, their energy increases or decreases respectively with decreasing frequency of the mode and vice versa for the holes. Hence, formation of a hole in the distribution function of trapped particles in the equilibrium field accompanies a clump in the distribution of passing ones and vice versa. The change in the perturbed potential energy of the trapped electrons in the BGK mode is relatively small compared to the change in their equilibrium energy when the change in $J_{\text {res }, \alpha}(t)$ is greater than the change in the separatrix width (see Appendix $\mathrm{C}$ for more details). More energy is released by the fast particles via the motion of the phase-space structures than in the process of their formation and the released energy during chirping should compensate the dissipated energy into the bulk. The total amount of power released corresponding to the change of the structure energy is given by

$$
P_{r}=-\sum_{\alpha} N_{\alpha} \frac{\mathrm{d} E_{\alpha}}{\mathrm{d} t}
$$

where $N_{\alpha}$ is the total number of each group of electrons in the hole/clump, $\frac{\mathrm{d} E_{\alpha}}{\mathrm{d} t}=\Omega_{\alpha}\left(\frac{\mathrm{d} \Omega_{\alpha}}{\mathrm{d} J_{\alpha}}\right)^{-1} \frac{\mathrm{d} \omega(t)}{\mathrm{d} t}$ is the rate of change of the energy of each particle and the resonance condition allows setting $\Omega_{\alpha}=\omega(t)$. Regarding to the definition of the adiabatic invariant of the trapped particles, $N_{\alpha}$ can be calculated as

$N_{\alpha}=\frac{2}{m_{e}}\left[F_{\mathrm{eq}, \alpha}(t=0)-F_{\mathrm{eq}, \alpha}(t)\right]$

$$
\begin{aligned}
& \times \int_{0}^{2 \pi}\left[\left(U_{\alpha, \operatorname{ext}}-\frac{1}{2} \sum_{n} A_{n}(t) V_{\alpha, n, n}\right.\right. \\
& \left.\times \exp (i n \tilde{\theta})+c . c) \frac{2}{\Delta_{\alpha}}\right]^{\frac{1}{2}} d \tilde{\theta} .
\end{aligned}
$$

The work done by the collision force can be used to calculate the dissipated power $\left(P_{d}\right)$ into the bullk via collisions. Using the equation of motion (4c) and considering the collisional term, we have

$$
P_{d}=\frac{2 \pi \nu k_{p}}{\omega^{2} m_{e}}\left\langle U^{2}\right\rangle
$$

where \langle\rangle denotes averaging over one wavelength and $\left\langle U^{2}\right\rangle=\frac{1}{2} \sum_{n} A_{n}^{2}(t)$. The released power during the motion of the holes/clumps is equal to the power dissipated in the bulk through collisions. This power balance can be used to calculate the rate at which 
sweeping occurs, which results in

$$
\frac{\mathrm{d} \omega(t)}{\mathrm{d} t}=-\left[\frac{\nu n_{c} \pi k_{p}}{\omega^{3} m_{e}} \sum_{n} A_{n}^{2}(t)\right] \frac{1}{\sum_{\alpha} N_{\alpha}\left(\frac{\mathrm{d} \Omega_{\alpha}}{\mathrm{d} J_{\alpha}}\right)^{-1}} .
$$

\section{Numerical Scheme}

In this section, we first derive the equation of the mode structure at early stage of chirping, say $t_{0}$, considering only the contribution from the trapped electrons in the equilibrium magnetic field. It is worth noting that here the contribution of passing electrons in the magnetic field to the equations of early stage is arbitrarily neglected just for the purpose of normalization. A simple evaluation of equation (27) at initial phase of sweeping when $F_{\text {eq }, \alpha}(t=0)-F_{\text {eq }, \alpha}(t)=0$ and $\hat{\omega}=1$, demonstrates that only the first Fourier coefficient is non-zero (a sinusoidal mode structure) and is presented by

$A_{1,0}=-\left[\frac{\left.8 \omega_{\mathrm{pe}}^{2} \frac{\partial F_{\mathrm{eq}, \mathbf{T}}}{\partial \zeta_{\mathbf{T}}} \frac{\partial \zeta_{\mathbf{T}}}{\partial \hat{\omega}}\right|_{\hat{\omega}=1}}{3 \pi k_{p} n_{c} \sqrt{\left|\Delta_{\mathbf{T}, 0}\right|}}\right] V_{\mathbf{T}, 1,1,0} \sqrt{A_{1,0} V_{\mathbf{T}, 1,1,0}}$.

Here, we have used the subscript 0 to denote evaluation at $t=t_{0}$. The term $A_{1,0}$ can be expressed in terms of the linear growth rate to have

$$
A_{1,0}=\frac{16^{2} \gamma_{l}^{2}}{9\left|\Delta_{\mathbf{T}, 0}\right| V_{\mathbf{T}, 1,1,0} \pi^{4}} .
$$

We also let $\hat{A}_{n}(t)=A_{n}(t) / A_{1,0}, \hat{V}_{\alpha, n, n}(t)=$ $V_{\alpha, n, n}(t) / V_{\mathbf{T}, 1,1,0}, \quad \hat{\Gamma}_{\alpha}=\Delta_{\alpha} /\left|\Delta_{\mathbf{T}, 0}\right|, \quad \hat{U}_{\alpha, \mathrm{ext}}=$ $U_{\alpha, \text { ext }} / A_{1,0} V_{\mathbf{T}, 1,1,0}$ and $F_{\text {eq }, \alpha}(t)=c_{\alpha} \zeta_{\alpha}(t)$.

Normalizing equation (27) with respect to $A_{1,0}$ results in

$$
\begin{gathered}
\hat{A}_{n}(t)=\left[\frac{-3 \hat{\omega}^{2}}{\left.8 c_{\mathbf{T}} \frac{\partial \zeta_{\mathbf{T}}}{\partial \hat{\omega}}\right|_{\hat{\omega}=1}\left(n^{2} \hat{\omega}^{2}-1\right)}\right] \sum_{\alpha} c_{\alpha}\left[\zeta_{\alpha, 0}-\zeta_{\alpha}\right] \\
\times \int_{0}^{2 \pi}\left[\left(\hat{U}_{\alpha, \operatorname{ext}}-\frac{1}{2} \sum_{n} \hat{A}_{n}(t) \hat{V}_{\alpha, n, n} \exp (i n \tilde{\theta})\right.\right. \\
\left.\quad+\text { c.c) } \frac{2}{\hat{\Gamma}_{\alpha}}\right]^{\frac{1}{2}}\left[\hat{V}_{\alpha, n, n}(t) \exp (i n \tilde{\theta})+c . c\right] d \tilde{\theta},
\end{gathered}
$$

which can be solved iteratively to derive the Fourier coefficients. In order to avoid the singularity in the numerical approach, a special treatment is applied to the first coefficient when the values of $\omega$ are close to $\omega_{\mathrm{pe}}$. In this case, $\zeta_{\alpha}(t)$ can be linear-approximated around the initial plasma frequency to cancel the effect of the pole in the denominator of equation (34).
Likewise, differential equation (31) can be investigated for the early phase of the structures motion in phase-space considering only the effect of trapped particles in the magnetic field. Substituting expression (29) into differential equation (31) and using equations (15) and (33), one finds

$$
\frac{\mathrm{d}}{\mathrm{d} t} \frac{\left(\omega-\omega_{\mathrm{pe}}\right)^{2}}{\omega_{\mathrm{pe}}^{2}}=\frac{\nu}{3}\left(\frac{16 \gamma_{l}}{3 \pi^{2} \omega_{\mathrm{pe}}}\right)^{2} .
$$

We define the dimensionless time $\tau=\frac{\nu}{3}\left(\frac{16 \gamma_{l}}{3 \pi^{2} \omega_{\mathrm{pe}}}\right)^{2} t$ and multiply differential equation (31) by $\frac{3}{\nu\left(\frac{16 \gamma_{l}}{3 \pi^{2} \omega_{\mathrm{pe}}}\right)^{2}}$ to have

$$
\begin{aligned}
\frac{\mathrm{d} \hat{\omega}}{\mathrm{d} \tau}= & -\left[\frac{4}{\hat{\omega}^{3}}\right] \frac{\hat{C}_{\mathbf{T}}\left|\left(\frac{\mathrm{d} \hat{\omega}}{\mathrm{d} \zeta}\right)^{-1}\right|}{\left.\sum_{\alpha} \operatorname{sgn}_{\alpha}\right|_{\zeta=\zeta \text { resonance }}\left[\zeta_{\alpha, 0}-\zeta_{\alpha}\right]} \\
& \times\left\{\int _ { 0 } ^ { 2 \pi } \left[\left(\hat{U}_{\alpha, \text { ext }}-\sum_{n} \frac{\hat{A}_{n}}{2} \hat{V}_{\alpha, n, n}\right.\right.\right. \\
& \left.\left.\times \exp (i n \tilde{\theta})+c . c) \frac{2}{\hat{\Gamma}_{\alpha}^{3}}\right]^{\frac{1}{2}} d \tilde{\theta}\right\}^{-1}
\end{aligned}
$$

where $\operatorname{sgn}_{\alpha}$ is -1 and 1 for $\alpha=\mathbf{T}$ and $\mathbf{P}$, respectively. The above equation can be solved by a fourth-order Runge-Kutta method along with the iterative method used for solving the Fourier coefficients on the RHS.

In case that the electrons have small enough pitch angles (deeply passing electrons with $\zeta \gg 1$ ), their motion will not be affected by the equilibrium magnetic field and they move freely. In other words, $\theta=k_{\mathrm{eq}} z$ (see figure $5(b)$ for $\zeta=2$ ). Subsequently, only one resonance is non-zero and the orbit averaged mode amplitude is equal to unity (see figure $4(b)$ ) under this condition. In this high energy range, one can find that $k_{p} z=p \theta$ in the linear theory limit. Canonical equations of motion assure $\theta=\Omega_{\alpha=\mathbf{P}} t$ so using equation (12), the resonance condition becomes $\omega=k_{p} v$, where $v$ is the particle velocity. Consequently, solving equation (34) and differential equation (36) in the limit that $\zeta \gg 1$, reproduces exactly the same results as in [21], which serves as the benchmark of the code and the numerical approach.

\section{Results}

For illustration, we have arbitrarily restricted attention to cases where $k_{\mathrm{p}}=k_{\mathrm{eq}}$. In the linear regime, the plasma mode will grow at different rates depending on the initial orbits of the electrons interacting with the mode. Figure 3 demonstrates that the linear growth rate decreases to zero in the limit of having resonance with the particles close to the separatrix of the equilibrium motion. 


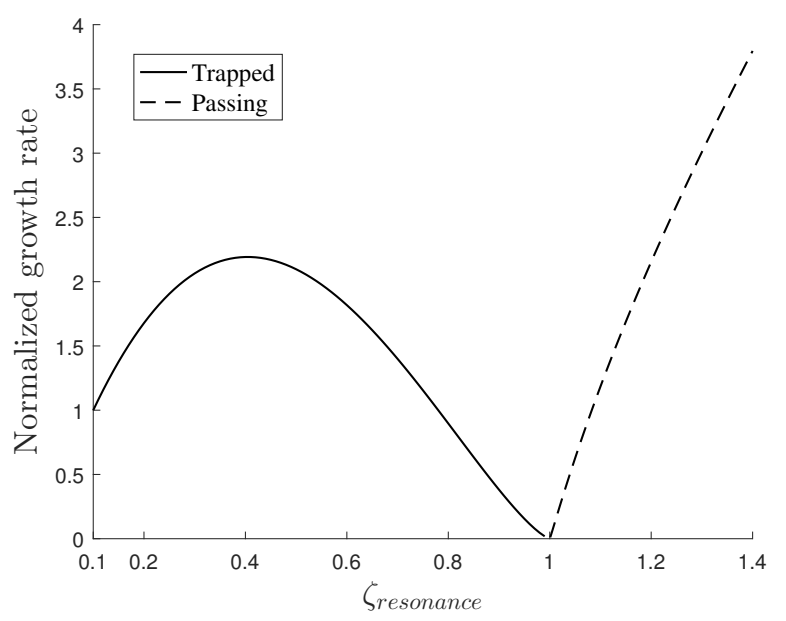

Figure 3. The linear growth rate behavior, corresponding to the first resonance, for different energy ranges of trapped and passing particles in the equilibrium magnetic field. All the values are normalized to the value at $\zeta=0.1$.

As in subsection 2.3 , the first resonance $(l=1)$ is considered as the dominant resonance contributing to the interaction. The first four elements of the orbit averaged mode amplitude $\hat{V}_{\alpha, n, p}$, indicating the coupling strength, corresponding to the first $\left(\hat{V}_{\alpha, n, n}\right)$ and the second $\left(\hat{V}_{\alpha, n, 2 n}\right)$ resonances are plotted

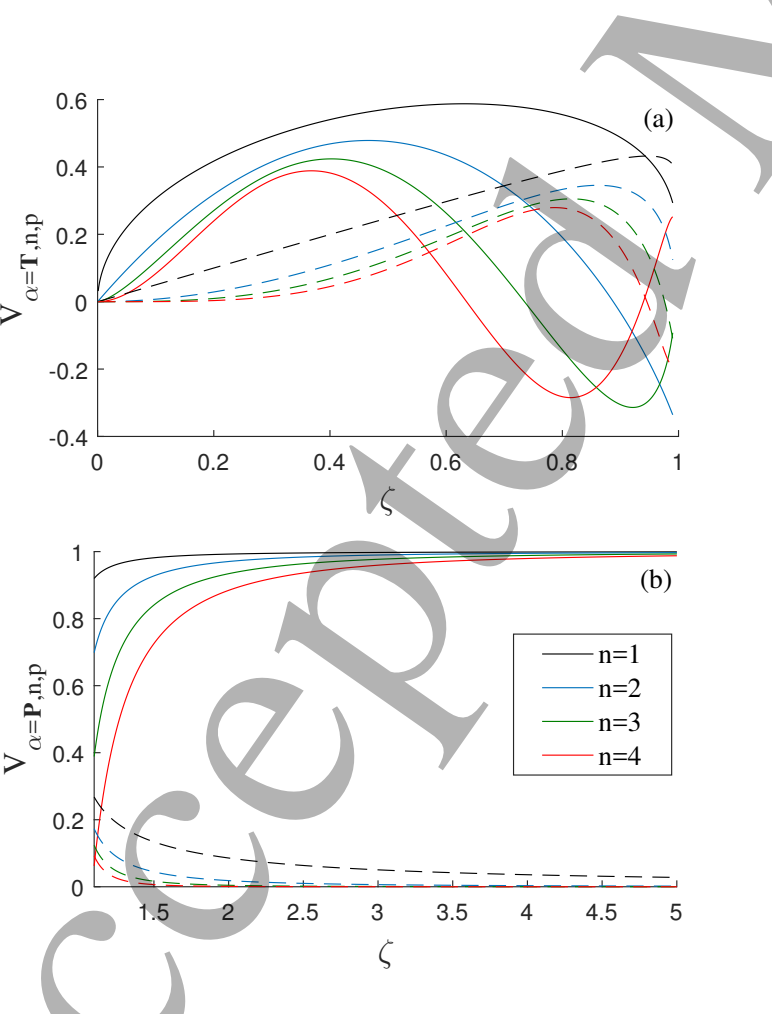

Figure 4. The orbit averaged mode amplitude versus energy parameter for (a) the trapped and (b) passing electrons in the equilibrium magnetic field. The solid and dashed lines correspond to first and second resonances, respectively.

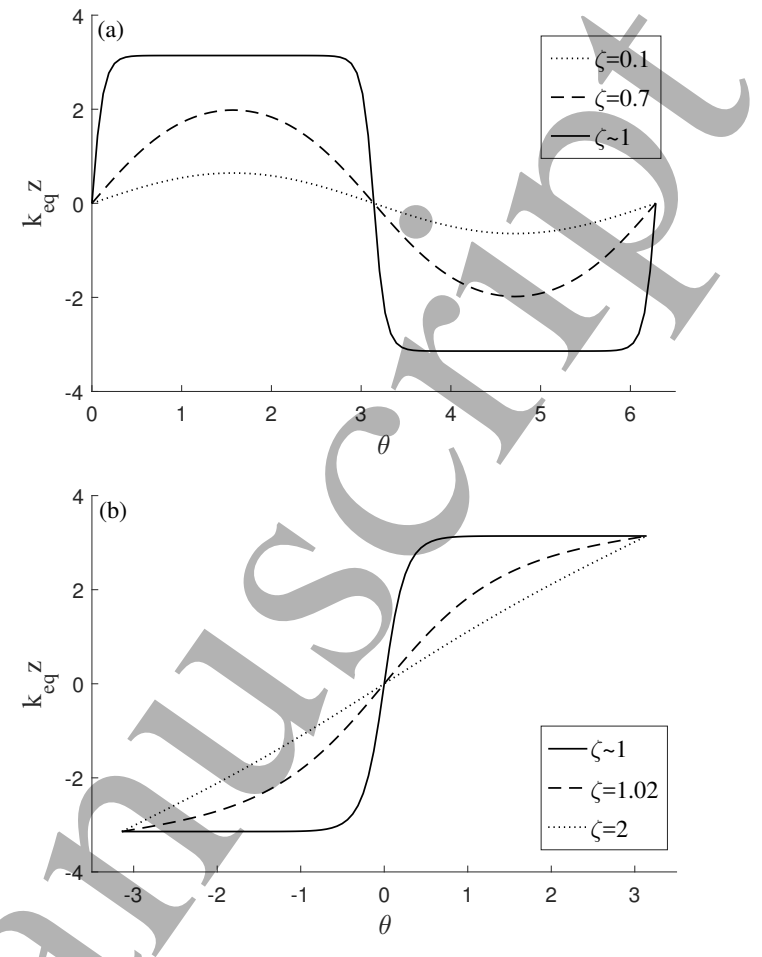

Figure 5. The position of energetic electrons (a) trapped and (b) passing in the equilibrium magnetic field in terms of the angle variable

in figure 4 versus energy parameter by numerical integrating of equation (9) over $\theta$. Investigation of figure 4 shows that there are regions (adjacent to $\zeta=1$ ) where the values of the dominant element $(n=1)$ belonging to the second resonance overtake the values of the dominant element of the first resonance. In itself, this may indicate that the corresponding second resonance is dominant. However, consideration of the linear growth rate for different resonances shows that the first resonant $(p=1)$ is dominant. This can be understood by inspection of equation (15): the term $\left|\frac{d \Omega_{\alpha}}{d \zeta_{\alpha}}\right|$ increases with increasing the resonance, so $\gamma_{l}$ decreases with increasing resonance. In addition, evaluating the factors of equation (34) for higher resonances $(l \geq 2)$ shows that its always the first resonance $(l=1)$ that has dominant contribution to the interaction in the hard nonlinear regime. Therefore, the submissive resonances are neglected. The other important point concerning the coupling strength is that all of its elements go asymptotically to zero as the energy parameter of the electrons approaches unity. Here, we explain this phenomenon in more detail: the equations (A.6) and (A.12) describe the equilibrium position $(z)$ of the electrons in terms of the action-angle variables in the nonuniform magnetic field. Figure 5 illustrates this position at different times for different energy parameters. For the case of trapped 

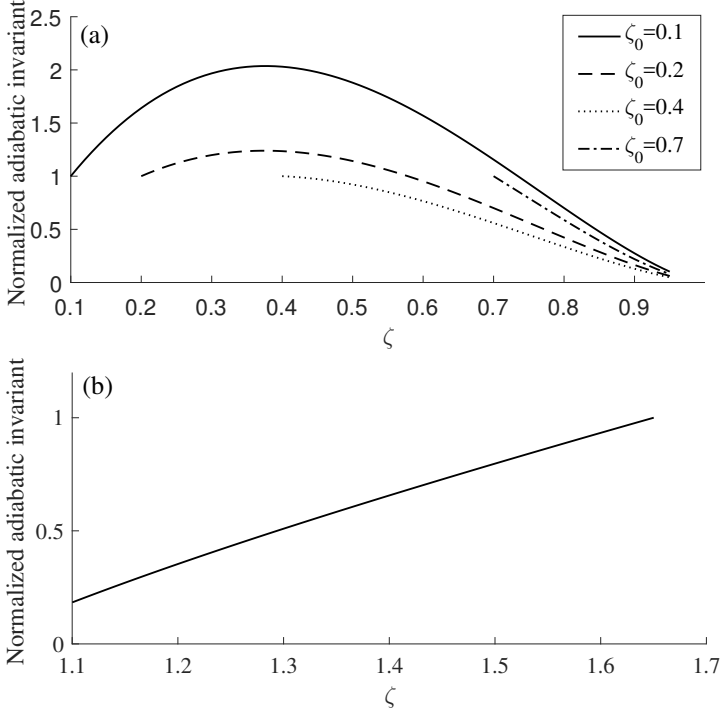

Figure 6. The values of the adiabatic invariant of (a) trapped and (b) passing particles in the equilibrium magnetic field for $k_{p} / k_{\text {eq }}=1$ at the separatrix determined by the BGK mode. The values are normalized with respect to the values at initial phase of chirping.

(figure 5(a)) and passing (figure $5(b)$ ) electrons, it is shown that for $\zeta \approx 1$, the electrons spend most of their period lingering at the two ends of the magnetic mirror system (the so-called magnetic bottle). This means that $z$ is almost $-\pi / k_{\text {eq }}$ during a half of the period and is almost $\pi / k_{\text {eq }}$ in the other half. Therefore, $\exp \left(i n k_{p} z\right)$ in the integrand of equation (9) is $\exp \left(i n k_{p} \pi / k_{\text {eq }}\right)$ or $\exp \left(-i n k_{p} \pi / k_{\text {eq }}\right)$ in each half period. For $k_{p} / k_{\text {eq }}=$ $m$ with $m$ an integer, we have $\exp \left(i n k_{p} \pi / k_{\text {eq }}\right)=$ $\exp \left(-i n k_{p} \pi / k_{\mathrm{eq}}\right)=$ cte and consequently the value of the integral drops to zero as the energy parameter approaches one $(\zeta \approx 1)$. These electrons barely move in $z$-direction, similar to the case where the electrons are deeply trapped $(\zeta \approx 0)$.

Prior to solving the equations for the mode structure and the sweeping rate in the hard nonlinear regime, it is necessary to investigate the behavior of the adiabatic invariant (phase-space area) of the trapped electrons in the BGK mode that are trapped or passing in the equilibrium magnetic field. Figure 6 shows the values of the adiabatic invariant (equation (22)) at the separatrix determined by the BGK mode during frequency sweeping. For the case of downward frequency sweeping, the energy of the passing electrons in the equilibrium field decreases, so does the corresponding value of the adiabatic invariant (figure $6(b)$ ). However, for trapped electrons, energy increases for downward frequency sweeping. Depending on the initial orbit, the adiabatic invariant can either initially increase $(\zeta<0.4$ of figure $6(a))$ or decrease $(\zeta \geq 0.4$ of figure $6(a))$. Due to the assumption of a flat-top distribution function over the separatrix region, the model remains valid as long as the separatrix supported by the BGK mode shrinks and an expanding separatrix (an increasing adiabatic invariant) should be avoided. Therefore, the electrons in the following results have initial energy parameters $\zeta \geq 0.4$. In this range, the coherent phase-space structure is a hole whose separatrix area (and the correspoding amplitude of the mode) is shrinking for a downsweeping frequency. For the case that new electrons are trapped into an expanding separatrix, it is required that the value of the distribution function of newly trapped particles is set to the value of the ambient distribution. The latter case is not the subject of this paper and the reader is referred to $[31,29,22]$ where the subject of expanding separatrices are addressed.

\subsection{The mode structure}

Considering similar slopes for the initial distribution of both the trapped and passing electrons in the equilibrium magnetic field (simultaneously in resonance with the plasma mode), the structure of the BGK mode has been solved for different initial electron energy parameters, namely $\zeta_{\alpha=\mathbf{T}}(t=0)=0.4$, 0.6 and 0.8. Figure 7 illustrates the mode structure for these initial energies in cases where $\hat{\omega}=0.8$ and 0.6 , constructed by solving equation (34) iteratively for the Fourier coefficients. The results reveal that for a nonzero change in $\hat{\omega}$, the nonlinear behavior of the BGK mode is determined by the initial electron orbits. For constant $\hat{\omega}$, e.g. figures $7(a),(c)$ and $(e)$, the maximum amplitude of the mode structure (maximum value of $\sum_{n} \hat{A}_{n} \cos \left(n k_{p} z\right)$ ) changes with changing $\zeta_{\alpha=\mathbf{T}, 0}$, and the change in the mode amplitude decreases with increasing $\zeta_{\alpha=\mathbf{T}, 0}$. The shape of the nonlinear structure is not only affected by the amount of change in the frequency $(\hat{\omega})$ but also by the initial energy parameter $\left(\zeta_{\alpha=\mathbf{T}, 0}\right)$. In order to explain the observed behavior, we first calculate the contribution of the trapped and passing particles to the mode structure seperately while they are simultaneously in resonance with the mode. Afterwards, the behavior of both the equilibrium frequency and the physical quantities appearing in equation (34) is investigated.

The Fourier coefficients are calculated by adding the two terms on the RHS of equation (34), corresponding to $\alpha=\mathbf{T}$ and $\mathbf{P}$. The seperate contributions of these two groups of particles to the mode structure are shown in figure 8 for similar values of distribution function and in case of simultaneous resonance between the plasma mode and these two types of energetic particles. It is clear that the contribution of the passing electrons to the nonlinear behavior of the mode is relatively much smaller than 
2

3
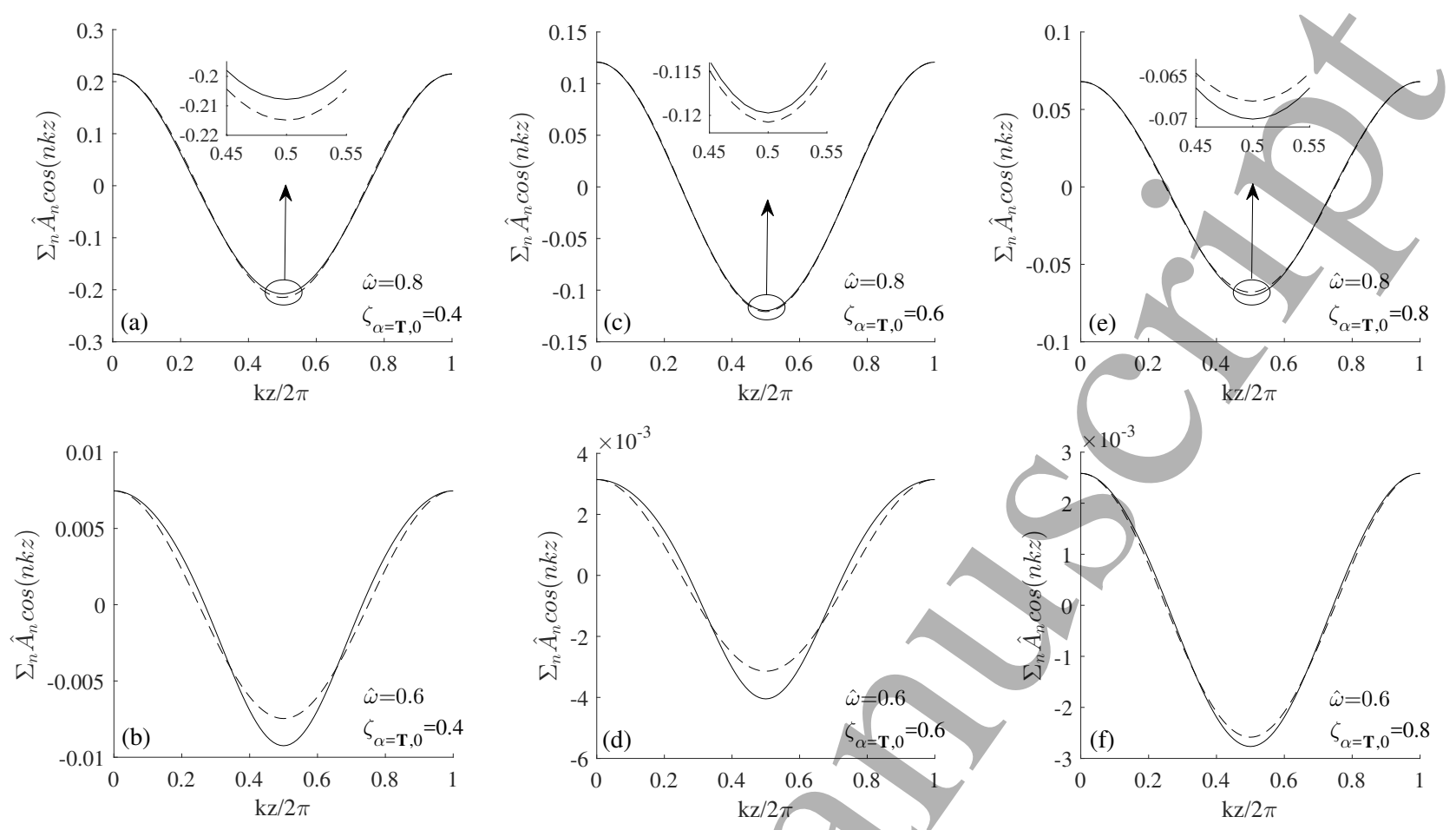

Figure 7. The normalized BGK mode structure affected by electrons having different initial energies. The dashed line, included here for comparison, represents the sinusoidal structure of the mode at early stage of frequency sweeping.

the trapped ones. The reason being that the resonance

At a constant value of the normalized frequency occurs in a region where the equilibrium frequency $\hat{\omega}$, a simple evaluation of equation (34) gives of passing particles has much steeper gradient in energy (see figure 1). Therefore, for the purpose of investigating the parameters of equation (34), we only consider the dominant contribution from the trapped electrons in the equilibrium magnetic field.

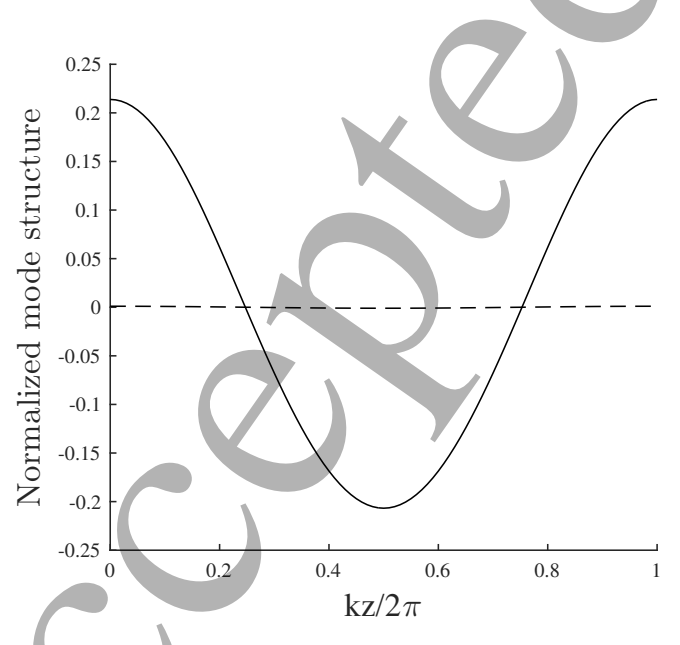

Figure 8. The contribution of trapped (solid line) and passing (dashed curve) electrons to the mode structure, where $\hat{\omega}=0.8$ and $\zeta_{\alpha=\mathbf{T}, 0}=0.4$. plotting on the same chart results in the small contribution of passing electrons to appear as a horizontal $\hat{A}_{n}(t) \propto \frac{\left(\frac{\mathrm{d} \hat{\omega}}{\mathrm{d} \zeta}\right)_{\alpha=\mathbf{T}, 0}^{2}\left[\zeta_{\alpha=\mathbf{T}}(t=0)-\zeta_{\alpha=\mathbf{T}}(t)\right]^{2} \hat{V}_{\alpha=\mathbf{T}, n, n}^{3}}{\hat{\Gamma}_{\alpha=\mathbf{T}}}$

Starting from different initial energies, the trapped electrons in the equilibrium magnetic field should be moved on different energy increments by the nonlinear mode in order to have the same amount of change in the frequency. This results from the nonlinear dependency of the equilibrium frequency on the energy parameter (see figure 1). As an example for $\hat{\omega}=0.8$, the fast electrons having the initial energy parameters of $\zeta_{\alpha=\mathbf{T}, 0}=0.4,0.6$ and 0.8 should be moved in phasespace to the points where $\zeta(t)=0.783,0.863$ and 0.94 , respectively and the energy increments become shorter for higher values of initial energy parameter. For a linear equilibrium distribution, the difference in the energy increments will explicitly appear in the nominator of equation (34) through the perturbed density term, i.e. $[\zeta(t=0)-\zeta(t)]$. In general, the nonlinear dependency of the equilibiurm frequency on the energy parameter will affect the values of all the physical parameters apearing in equation (34) for a fixed amount of frequency shift. Figure 9 shows the dependency of the factors $\left(\frac{\mathrm{d} \hat{\omega}}{\mathrm{d} \zeta}\right)_{\alpha=\mathbf{T}, 0}^{2}[\zeta(t=0)-\zeta(t)]^{2}$, 

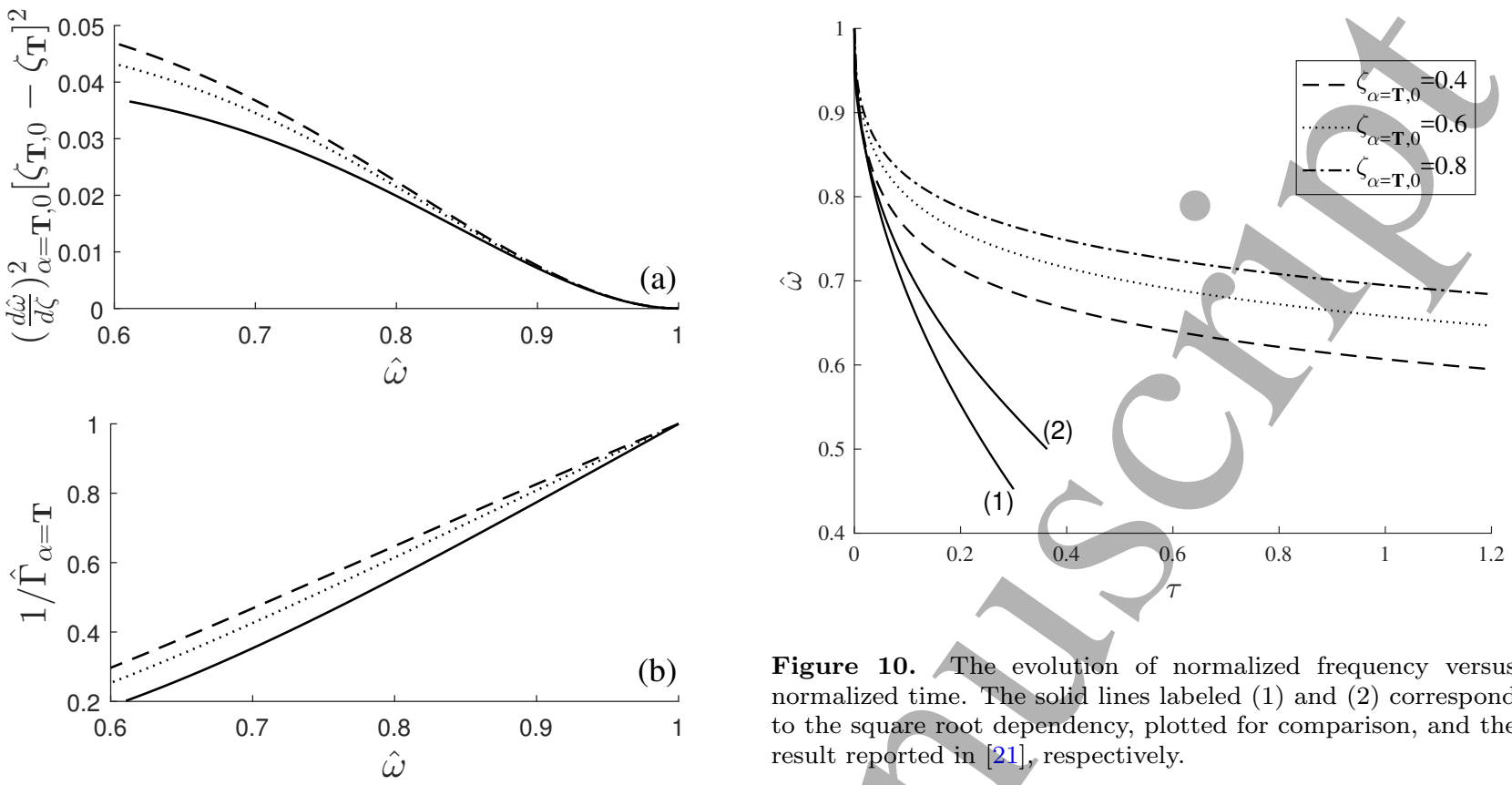

Figure 10. The evolution of normalized frequency versus normalized time. The solid lines labeled (1) and (2) correspond to the square root dependency, plotted for comparison, and the result reported in [21], respectively.

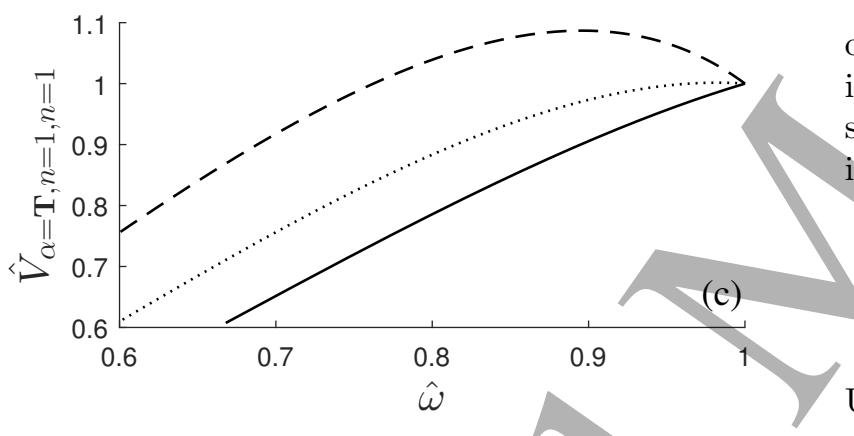

orbits) using the behavior of the factors illustrated in figure 9. Looking at the expression (36) for the sweeping rate at a constant value of $\hat{\omega}$, it can be inferred that

$$
\frac{\mathrm{d} \hat{\omega}}{\mathrm{d} \tau} \propto \frac{\hat{A}_{n}(t)^{\frac{3}{2}} \hat{\Gamma}_{\alpha=\mathbf{T}}^{\frac{3}{2}}}{\left|\left(\frac{\mathrm{d} \hat{\omega}}{\mathrm{d} \tau}\right)_{\alpha=\mathbf{T}, 0}\right|\left[\zeta_{\alpha=\mathbf{T}}(t=0)-\zeta_{\alpha=\mathbf{T}}(t)\right] \hat{V}_{\alpha=\mathbf{T}, n, n}^{\frac{1}{2}}} .
$$

Using expression (37) one finds

Figure 9. The factors in (37) versus the normalized frequency $(\hat{\omega})$. The dashed, dotted and solid lines correspond to the initial energy parameter $\left(\zeta_{\alpha=\mathbf{T}, 0}\right)$ values of $0.4,0.6$ and 0.8 , respectively.

$\hat{V}_{\alpha=\mathbf{T}, n=1, n=1}^{3}$ and $\hat{\Gamma}_{\alpha=\mathbf{T}}^{-1}$ in expression (37) for different $\zeta_{\alpha=\mathbf{T}, 0}$ and as a function of $\hat{\omega}$. The dependency of $\hat{A}_{n}$ with $\zeta_{\alpha=\mathbf{T}, 0}$ can be understood by inspection of these factors. At each $\hat{\omega}$ the factors decreases with increasing $\zeta_{\alpha=\mathbf{T}, 0}$ and so $\hat{A}_{n}$ decreases. It is noteworthy that $\left(\frac{\mathrm{d} \hat{\omega}}{\mathrm{d} \zeta}\right)_{\alpha=\mathbf{T}, 0}$ can be calculated by differentiating the normalized form of equation (8a) with respect to $\zeta$, which together with the differentiation of equation (5a) can be used to derive $\hat{\Gamma}_{\alpha=\mathbf{T}}$.

\subsection{The sweeping rate and adiabaticity validation}

In this subsection, we first investigate the rate at which the frequency of the nonlinear mode evolves in time. Prior to solving the differential equation (36), we evaluate the dependency of the sweeping rate $\left(\frac{d \hat{\omega}}{d \tau}\right)$ on the initial energy parameter of the electrons (initial

$$
\frac{\mathrm{d} \hat{\omega}}{\mathrm{d} \tau} \propto\left(\frac{\mathrm{d} \hat{\omega}}{\mathrm{d} \zeta}\right)_{\alpha=\mathbf{T}, 0}^{2}\left[\zeta_{\alpha=\mathbf{T}}(t=0)-\zeta_{\alpha=\mathbf{T}}(t)\right]^{2} \hat{V}_{\alpha=\mathbf{T}, n, n}^{4} .
$$

Similar to subsection 4.1 , one can consider figures $9(a)$ and $(c)$ at a constant $\hat{\omega}$ to investigate the value of the RHS of expression (39) for different electron orbits. It is clear that the RHS value becomes lower when the resonance occurs with the electrons (trapped in the fixed equilibrium magnetic field) having higher initial energy parameter $\left(\zeta_{\alpha=\mathbf{T}, 0}\right)$. Therefore, we expect the mode frequency to chirp slower when the initial energy parameter of the electrons is higher. This can be verified by solving differential equation (36) using the numerical method stated in section 3 for different initial orbits. Figure 10 illustrates the time evolution of $\hat{\omega}$ for different values of $\zeta_{\alpha=\mathbf{T}, 0}$. The results reproduce the square root dependency for initial stages of chirping as in $[11,21]$. However, it is shown that in this model, the holes and clumps can move with much lower rates compared with the sweeping rates observed in [21]. On the other hand, as predicted above, for higher initial energy parameter of the trapped electrons in the equilibrium magnetic field, the frequency tends to 

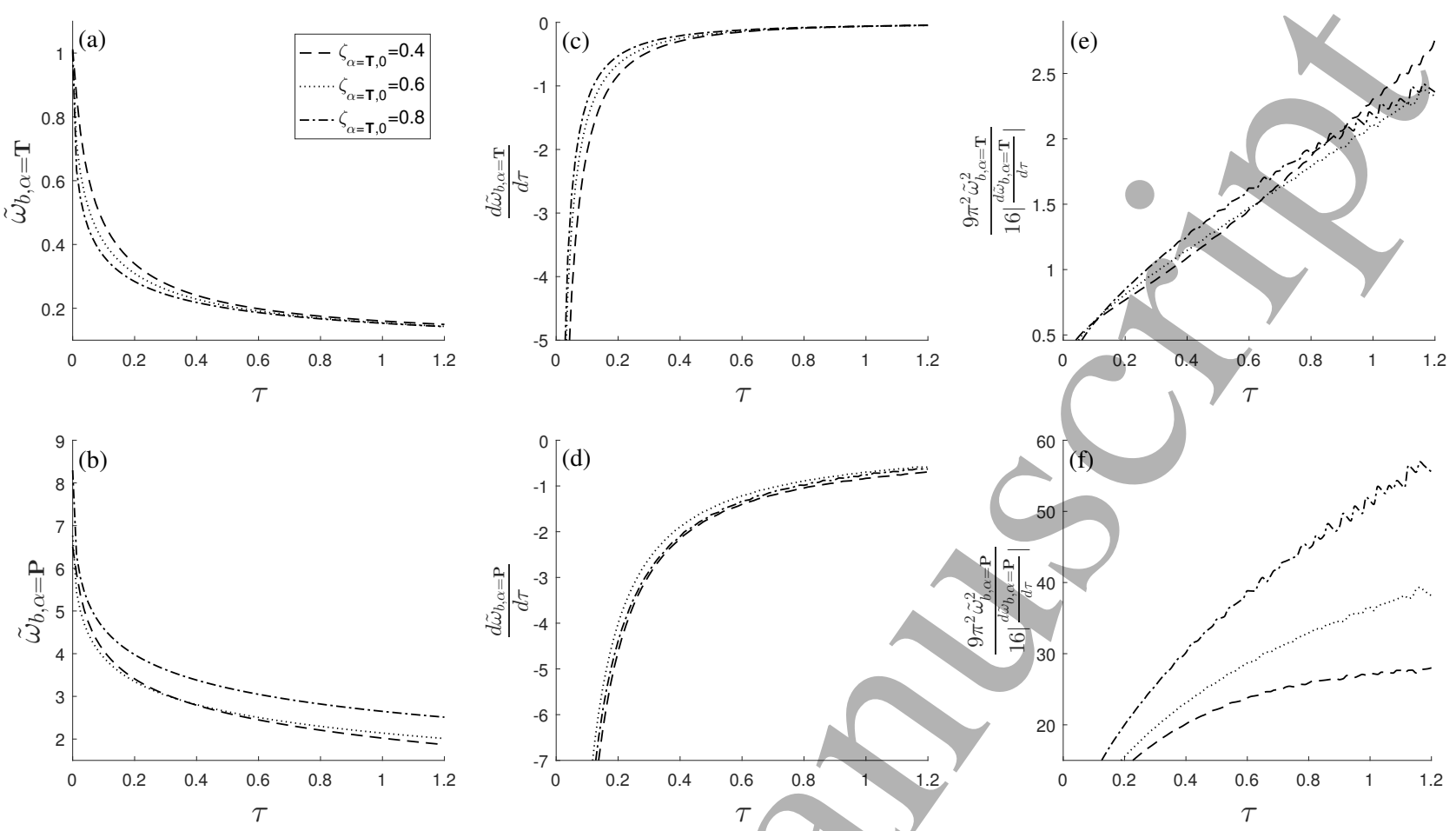

Figure 11. The evolution of the normalized bounce frequency $(\mathrm{a}, \mathrm{b})$, normalized time rate of change in the bounce frequency (c,d) and the value of the RHS of inequality $(45)(e, f)$. Panels $(a, c, e)$ and $(b, d, f)$ correspond to trapped and passing electrons in the magnetic field, respectively. The dashed, dotted and dash-dotted curves represent an initial energy parameter value of $0.4,0.6$ and 0.8 , respectively, for trapped electrons in the magnetic field. At $\tau=0$, the values of panels (c,d) go asymptotically to $-\infty$ and for panels $(e, f)$ the corresponding values are zero.

decrease more slowly.

As mentioned earlier in subsection 2.3, the adiabaticity condition invoked for the analysis, should be checked if it remains satisfied when the frequency deviates from the initial eigenfrequency. Using the total Hamiltonian of the resonant electrons (Equation (19)), the canonical equations of the perturbed motion of these electrons, read

$$
\begin{aligned}
& \dot{\tilde{J}}_{\alpha}=\sum_{n} A_{n} V_{\alpha, n, n} n \sin (n \tilde{\theta}), \\
& \dot{\tilde{\theta}}=\left.\frac{\partial^{2} H_{0, \alpha}}{\partial \tilde{J}_{\alpha}^{2}}\right|_{\tilde{J}_{\alpha}=J_{\mathrm{res}, \alpha}}\left(\tilde{J}_{\alpha}-J_{\mathrm{res}, \alpha}\right) .
\end{aligned}
$$

In the absence of collisions, the motion of resonant electrons which are trapped in the equilibrium field and are deeply trapped in the BGK mode satisfies the pendulum equation

with $\left|\Delta_{\alpha=\mathbf{T}} \sum_{n} A_{n} V_{\alpha=\mathbf{T}, n, n} n^{2}\right|=\omega_{b, \alpha=\mathbf{T}}^{2}$, where we have used $\sin (n \tilde{\theta}) \approx n \tilde{\theta}$ at the center of the separatrix, the so-called O-point which is at $\tilde{\theta}=0$ for trapped electrons in the magnetic field (see figure $2(b)$ ).
Similarly, for passing electrons in the magnetic field that are deeply trapped in the BGK mode, we have

$$
\begin{aligned}
\frac{\mathrm{d}^{2}}{\mathrm{~d} t^{2}}(\tilde{\theta}-\pi) & =\Delta_{\alpha=\mathbf{P}} \sum_{n} A_{n} V_{\alpha=\mathbf{P}, n, n} n^{2} \cos (n \pi) \\
& \times(\tilde{\theta}-\pi),
\end{aligned}
$$

with $\left|\Delta_{\alpha=\mathbf{P}} \sum_{n} A_{n} V_{\alpha=\mathbf{P}, n, n} n^{2} \cos (n \pi)\right|=\omega_{b, \alpha=\mathbf{P}}^{2}$, where we have expanded $\sin (n \tilde{\theta})$ about the O-point at $\tilde{\theta}=\pi$ for passing electrons in the magnetic field (see figure $2(a)$ ).

We introduce the dimensionless variable $\tilde{\omega}_{b, \alpha}=$ $\frac{\omega_{b, \alpha}}{\omega_{b, \alpha=\mathbf{T}, t=0}}$, with

$$
\omega_{b, \alpha=\mathbf{T}, t=0}=\sqrt{\left|A_{1, t=0} V_{\alpha=\mathbf{T}, n=1, n=1} \Delta_{\alpha=\mathbf{T}}\right|},
$$

the bounce frequency of resonant electrons (trapped in the magnetic field) in their corresponding separatrix in the BGK mode at early stage of chirping denoted by $t=0$. We can write the adiabatic limit, introduced in subsection 2.3 , in the form

$$
\frac{\frac{\mathrm{d} \tau}{\mathrm{d} t}}{\omega_{b, \alpha=\mathbf{T}, t=0}} \ll \frac{\tilde{\omega}_{b, \alpha}^{2}}{\left|\frac{\mathrm{d} \tilde{\omega}_{b, \alpha}}{\mathrm{d} \tau}\right|} .
$$


Using equation (33) and the expression for the dimensionless time introduced in section 3 , one finds

$$
\frac{\nu \gamma_{l, \alpha=\mathbf{T}}}{w_{\mathrm{pe}}^{2}} \ll \frac{9 \pi^{2} \tilde{\omega}_{b, \alpha}^{2}}{16\left|\frac{\mathrm{d} \tilde{\omega}_{b, \alpha}}{\mathrm{d} \tau}\right|} .
$$

The time evolution of $\tilde{\omega}_{b}$ can be investigated in the same numerical code implemented to solve differential equation (36) for constructing figure 10. At each time step in the fourth-order Runge-Kutta method, the corresponding parameters can be used to derive $\tilde{\omega}_{b, \alpha}(\tau)$. Afterwards, one can readily use numerical differentiation methods to find $\frac{\mathrm{d} \tilde{\omega}_{b, \alpha}}{\mathrm{d} \tau}$.

Figures 11(a) and (b) shows the normalized bounce frequency of the trapped electrons about the O-point of the separatrix inside the BGK mode corresponding to the trapped and passing electrons in the magnetic field, respectively, for the initial energy parameters considered in the previous subsections. The corresponding values of $\frac{\mathrm{d} \tilde{\omega}_{b, \alpha}}{\mathrm{d} \tau}$ are demonstrated in figures $11(c)$ and $(d)$, where the values decrease to $-\infty$ as we approach $\tau=0$. Therefore, the value of the RHS of (45), illustrated in figures $11(e)$ and $(f)$, drops to zero at the early stage of frequency chirping. This means that the adiabatic limit is never formally satisfield at initial stage of phase-space structures evolution. Nevertheless, we have $\gamma_{l}, \nu \ll \omega_{\mathrm{pe}}$ and as a result the period during which the adiabatic condition is not satisfied is extremely short. The results reveal that as the system evolves while the adiabaticity limit is initially violated, for later evolution of phasespace structures the RHS value of (45) monotonically increases. Therefore, once the adiabatie limit (45) is satisfied with regards to the value of LHS, it will remain valid for later evolution. It should be noted that the adiabaticity condition is better satisfied for the passing electrons in the magnetic field compared to the trapped ones.

\section{Concluding remarks}

The more realistic 1D model shows that apart from the amount of deviation from the initial eigenfrequency during frequency sweeping, the initial orbit (initial energy parameter) of the particles in a nonuniform equilibrium magnetic field, determines both the linear and the hard nonlinear evolution behavior of a plasma mode. The model also resolves the simultaneous contributions from the two groups of particles having different orbit types as well as the contribution from higher resonances. We find however that the first resonance is dominant. We also identify different behavior of the adiabatic invariant in different energy regions. The model shows that for a constant trend in frequency sweeping, either upward or downward, the adiabatic invariant can have both positive and negative gradients in the energy parameter depending on the energy region considered. This behavior depends on factors such as the resonance number, the proportion of the plasma mode wave-number to the spatial frequency of the equilibrium field $\left(k_{p} / k_{\text {eq }}\right)$ and whether the particles were initially trapped or passing in the equilibrium field. This indicates that for realistic geometries where particles interacting with the mode can follow different equilibrium orbits, an extended approach is required to calculate the perturbed density inside the holes and clumps. The required approach should take into account that the adiabatic invariant (phase-space area) at the separatrix can initially expand followed by a shrinking behavior and vice versa, depending on the initial orbit of the energetic particles. This extension can highly benefit from the method presented in [22].

The presented model in this manuscript provides a more effective understanding of hard nonlinear waveparticle-plasma interactions in realistic geometries provided that the mode is subject to weak continuum damping (a global mode) i.e. its structure in the linear regime is not mainly determined by the energetic particles. Two different orbit topologies of energetic particles created by adding a nonuniform magnetic field to the 1D bump-on-tail instability problem, bring it into anology with tokamaks where trapped and passing topologies exist which can both resonate with modes with different coupling strength factors. In a high aspect ratio tokamak, the total magnetic field follows

$$
B \propto \frac{1}{R_{0}+r \cos \theta} \propto \frac{1}{R_{0}}(1-\epsilon \cos \theta),
$$

where $B$ is the magnetic field, $\epsilon$ is the inverse aspect ratio, $\theta$ is the poloidal angle and $R_{0}$ and $r$ are the major and minor radius, respectively.

Using the orbit-averaged Littlejohn's Hamiltonian, we have

$$
H_{0}-\mu B_{0}=\frac{1}{2} m_{i} v_{\|}^{2}-\mu B_{0} \epsilon \cos (\theta),
$$

where $H_{0}$ is the equilibrium Hamiltonian and $v_{\|}$is the velocity in the direction of the magnetic field. Taking into account the symmetry of the magnetic field in toroidal direction in realistic geometries and assuming that the deviation of the fast particles from the flux surface is infinitesimal, the above Hamiltonian is comparable to the equilibrium Hamiltonian presented in equation (3). Further restrictions on the perturbation such as symmetry in toroidal direction, being localized on one flux surface and the assumption that the perturbation on different flux surfaces are unlinked, might let the presented model to describe some features of electrostatic axisymmetric modes 
( $n=0$, where $\mathrm{n}$ is the poloidal mode number), namely global geodesic acoustic modes (GGAMs) in more realistic geometries [32]. Nevertheless, an exact description of excited Global-Alfven-Eigenmodes (GAEs) with an evolving mode structure during long range frequency deviations requires the extension of the presented model, which is a part of our ongoing research. Another avenue for further research is to relax the assumption that the fast electron distribution function is linear.

\section{Acknowledgments}

The authors wish to gratefully thank Prof. Boris Breizman from Institute for Fusion Studies, The University of Texas at Austin, USA for stimulating discussions that helped inspire this paper. The authors are grateful to Prof. Robert L. Dewar from Research School of Physics and Engineering, The Australian National University, Canberra, Australia and Dr. Michael Fitzgerald from CCFE, Culham Science Centre, UK for useful comments and discussions. This work was funded by the Australian Research Council through Grant No. DP140100790.

\section{References}

[1] Chen L 1994 Physics of Plasmas 1 1519-1522

[2] Heidbrink W W 2008 Physics of Plasmas 15055501

[3] ITER Physics Expert Group on Energetic Particles H, Drive C and Editors I P B 1999 Nuclear Fusion 392471

[4] Fasoli A, Gormenzano C, Berk H, Breizman B, Briguglio S, Darrow D, Gorelenkov N, Heidbrink W, Jaun A, Konovalov S, Nazikian R, Noterdaeme J M, Sharapov S, Shinohara K, Testa D, Tobita K, Todo Y, Vlad G and Zonca F 2007 Nuclear Fusion 47 S264

[5] Heidbrink W and Sadler G 1994 Nuclear Fusion 34535

[6] Heidbrink W, Strait E, Doyle E, Sager G and Snider R 1991 Nuclear Fusion 311635

[7] Wong K L, Fonck R J, Paul S F, Roberts D R, Fredrickson E D, Nazikian R, Park H K, Bell M, Bretz N L, Budny R, Cohen S, Hammett G W, Jobes F C, Meade D M, Medley S S, Mueller D, Nagayama Y, Owens D K and Synakowski E J 1991 Phys. Rev. Lett. 66(14) 1874-1877

[8] Pinches S D, Berk H L, Gryaznevich M P, Sharapov S E and Contributors J E 2004 Plasma Physics and Controlled Fusion 46 S47

[9] Fredrickson E, Gorelenkov N, Bell R, Menard J, Roquemore A, Kubota S, Crocker N and Peebles W 2006 Nuclear Fusion 46 S926

[10] Shinohara K, Kusama Y, Takechi M, Morioka A, Ishikawa M, Oyama N, Tobita K, Ozeki T, Takeji S, Moriyama S, Fujita T, Oikawa T, Suzuki T, Nishitani T, Kondoh T, Lee S, Kuriyama M, Team J , Kramer G, Gorelenkov N, Nazikian R, Cheng C, Fu G and Fukuyama A 2001 Nuclear Fusion 41603

[11] Berk H, Breizman B and Petviashvili N 1997 Physics Letters A 234 213-218 ISSN 0375-9601

[12] Bernstein I B, Greene J M and Kruskal M D 1957 Phys. Rev. 108(3) 546-550

[13] Lilley MK and Nyqvist R M 2014 Phys. Rev. Lett. 112(15) 155002
[14] Fasoli A, Breizman B N, Borba D, Heeter R F, Pekker M S and Sharapov S E 1998 Phys. Rev. Lett. 81(25) 55645567

[15] Heeter R F, Fasoli A F and Sharapov S E 2000 Phys. Rev. Lett. 85(15) 3177-3180

[16] Lilley M K, Breizman B N and Sharapov S E 2009 Phys. Rev. Lett. 102(19) 195003

[17] Lilley M K, Breizman B N and Sharapov S E 2010 Physics of Plasmas 17092305

[18] Gryaznevich M and Sharapov S 2000 Nuclear Fusion 40 907

[19] Maslovsky D, Levitt B and Mauel M E 2003 Phys. Rev. Lett. 90(18) 185001

[20] Fredrickson E D, Bell R E, Darrow D S, Fu G Y, Gorelenkov N N, LeBlanc B P, Medley S S, Menard J E, Park H, Roquemore A L, Heidbrink W W, Sabbagh S A, Stutman D, Tritz K, Crocker N A, Kubota S, Peebles W, Lee K C and Levinton F M 2006 Physics of Plasmas 13056109

[21] Breizman B N 2010 Nuclear Fusion 50084014

[22] Nyqvist R M and Breizman B N 2013 Physics of Plasmas 20042106

[23] Littlejohn R G 1983 Journal of Plasma Physics 29 111-125

[24] White R B 2006 The Theory of Toroidally Confined Plasmas 2nd ed (London: Imperial College Press)

[25] Berk H, Breizman B and Pekker M 1995 Nuclear Fusion 35 1713

[26] Breizman B N, Berk H L, Pekker M S, Porcelli F, Stupakov G V and Wong K L 1997 Physics of Plasmas 4 15591568

[27] Nyqvist R, Lilley M and Breizman B 2012 Nuclear Fusion 52094020

[28] Berk H L, Breizman B N, Candy J, Pekker M and Petviashvili N V 1999 Physics of Plasmas 6 3102-3113

[29] Eremin D Y and Berk H L 2002 Physics of Plasmas 9 772785

[30] Wang G and Berk H 2012 Nuclear Fusion 52094003

[31] Wang G and Berk H 2012 Communications in Nonlinear Science and Numerical Simulation 17 2179-2190 ISSN 1007-5704 special Issue: Mathematical Structure of Fluids and PlasmasDedicated to the 60th birthday of Phil Morrison

[32] Berk H, Boswell C, Borba D, Figueiredo A, Johnson T, Nave M, Pinches S, Sharapov S and contributors J E 2006 Nuclear Fusion 46 S888 
Appendix A. Calculation of $z(J, \theta)$

Using the equilibrium Hamiltonian (3) and $p_{z}=m_{e} \frac{\mathrm{d} z}{\mathrm{~d} t}$, we find

$$
\frac{2}{k_{\text {eq }}} \frac{\mathrm{d} \sigma}{\mathrm{d} t}=\sqrt{\frac{2}{m_{e}}\left[E+\mu\left(B_{c}+B_{0} \cos (2 \sigma)\right)\right]}
$$

where $\sigma=\frac{k_{\mathrm{eq}} z}{2}$. We take $\sigma(t=0)=0$, to have

$\frac{\sqrt{2} k_{\mathrm{eq}}^{-1}}{\sqrt{\frac{E+\mu\left(B_{0}-B_{c}\right)}{m_{e}}}} \int_{0}^{\sigma} \frac{d \sigma}{\sqrt{1-\frac{2 \mu B_{0}}{E+\mu\left(B_{0}-B_{c}\right)} \sin ^{2}(\sigma)}}=\int_{0}^{t} d t$

where we have used $\cos (2 \sigma)=1-2 \sin ^{2} \sigma$.

I) For passing electrons in the nonuniform magnetic field, the coefficient of $\sin ^{2}(\sigma)$ in integral equation (A.2) is less than unity. After changing the coordinates to action-angle variables in subsection 2.1, we can use the canonical equations of motion to find

$$
t=\frac{\theta}{\Omega_{\mathrm{eq}, \alpha}},
$$

where $\Omega_{\mathrm{eq}, \alpha=\mathbf{P}}$ is presented by equation (8b). Substituing (A.3) into the RHS of (A.2), we find

$$
\int_{0}^{\sigma} \frac{d \sigma}{\sqrt{1-\frac{2 \mu B_{0}}{E+\mu\left(B_{0}-B_{c}\right)} \sin ^{2}(\sigma)}}=\frac{\theta \mathbb{K}\left(\zeta^{-1}\right)}{\pi} .
$$

According to the definition of Jacobi elliptic functions, we find

$$
\operatorname{Sn}\left(\frac{\theta \mathbb{K}\left(\zeta^{-1}\right)}{\pi}, \zeta^{-1}\right)=\sin \sigma
$$

which gives

$$
z_{\alpha=\mathbf{P}}=\frac{2}{k_{\mathrm{eq}}} \sin ^{-1}\left[\operatorname{Sn}\left(\frac{\theta \mathbb{K}\left(\zeta^{-1}\right)}{\pi}, \zeta^{-1}\right)\right]
$$

II) For trapped electrons in the nonuniform magnetic field, the coefficient of $\sin ^{2}(\sigma)$ in integral equation (A.2) is higher than unity. we implement a change of variables as follows,

$$
\begin{aligned}
& \sin (\eta)=\frac{\sin (\sigma)}{\sin \left(\sigma_{\max }\right)} \\
& d \sigma=\frac{\sin \left(\sigma_{\max }\right) \cos (\eta)}{\sqrt{1-\sin ^{2}\left(\sigma_{\max }\right) \sin ^{2}(\eta)}} d \eta
\end{aligned}
$$

The maximum value of $z$ is $\frac{1}{k_{\mathrm{eq}}} \cos ^{-1}\left(\frac{\mu B_{c}-E}{\mu B_{0}}\right)$, derived from $p_{z}=0$. Hence, $\cos \left(2 \sigma_{\max }\right)=\frac{\mu B_{c}-E}{\mu B_{0}}$ and

$$
\sin ^{2}\left(\sigma_{\max }\right)=\frac{E+\mu\left(B_{0}-B_{c}\right)}{2 \mu B_{0}} .
$$

Now we substitute equations (A.7a) and (A.7b) into (A.2) and use (A.8) to have

$$
\frac{\sqrt{2} \sin \left(\sigma_{\max }\right)}{k_{\mathrm{eq}} \sqrt{\frac{E+\mu\left(B_{0}-B_{c}\right)}{m_{e}}}} \int_{0}^{\eta} \frac{d \eta}{\sqrt{1-\sin ^{2}\left(\sigma_{\max }\right) \sin ^{2}(\eta)}}=t,
$$

where we have used $\frac{\cos \eta}{\sqrt{1-\frac{2 \mu B_{0}}{E+\mu\left(B_{0}-B_{c}\right)} \sin ^{2}(\sigma)}}=1$. Using equation (A.3) for trapped electrons in the magnetic field and equation (8b), we find

$$
\int_{0}^{\eta} \frac{d \eta}{\sqrt{1-\sin ^{2}\left(\sigma_{\max }\right) \sin ^{2}(\eta)}}=\frac{2 \theta \mathbb{K}(\zeta)}{\pi},
$$

which gives

$$
\operatorname{Sn}\left(\frac{2 \theta \mathbb{K}(\zeta)}{\pi}, \zeta\right)=\sin \eta=\frac{\sin \sigma}{\sin \sigma_{\max }} .
$$

We find

$$
z_{\alpha=\mathbf{T}}=\frac{2}{k_{\mathrm{eq}}} \sin ^{-1}\left[\sqrt{\zeta} \operatorname{Sn}\left(\frac{2 \theta \mathbb{K}(\zeta)}{\pi}, \zeta\right)\right] .
$$

It should be mentioned that equations (A.6) and (A.12) can be inverted for the corresponding angle $\theta(z, \zeta)$ variables.

\section{Appendix B. Adiabatic invariant and bounce averaging method}

The adiabatic invariant for a Hamiltonian $K(\hat{\theta}, \hat{J}, \lambda \equiv$ $\beta t)$ with slow time dependency $(\beta \ll$ typical orbit frequencies) is

$$
I^{\infty}=I(q, p, \lambda)+\beta I_{1}(q, p, \lambda)+\beta^{2} I_{2}(q, p, \lambda)+\ldots,
$$

which the lowest term is commonly taken to be the action, $I(E, \lambda)=\oint \hat{J}(\hat{\theta}, E, \lambda) d \hat{\theta}$ with $K(\hat{\theta}, \hat{J}, \lambda)=E$. We transform to action-angle variables using the generating function $\Phi_{2}(\hat{\theta}, I, \lambda)=$ $\int_{\hat{\theta}_{0}(I, \lambda)}^{\hat{\theta}} d \hat{\theta}^{\prime} \hat{J}\left(\hat{\theta}^{\prime}, K(I, \lambda), \lambda\right)$. So the Hamiltonian transforms into $K_{\text {new }}(\Theta, I, \lambda)=K(I, \lambda)+\beta \frac{\partial \Phi_{2}}{\partial \lambda}$. Now we consider the trapped electron Vlasov equation

$$
\frac{\partial f}{\partial t}+\frac{\partial f}{\partial \Theta} \frac{\partial K_{n e w}}{\partial I}-\frac{\partial f}{\partial I} \frac{\partial K_{n e w}}{\partial \Theta}=0 .
$$

Using the equations of motion we have

$$
\begin{aligned}
& \dot{\Theta}=\frac{\partial K_{\text {new }}}{\partial I}=\omega_{\text {Bounce }}+\frac{\partial}{\partial I} \frac{\partial \Phi_{2}}{\partial t}, \\
& \dot{I}=-\frac{\partial K_{\text {new }}}{\partial \Theta}=-\frac{\partial}{\partial \Theta} \frac{\partial \Phi_{2}}{\partial t},
\end{aligned}
$$


Substituting the above expressions in equation (B.2) gives

$$
\frac{\partial f}{\partial t}+\frac{\partial f}{\partial \Theta} \omega_{\text {Bounce }}+\frac{\partial f}{\partial \Theta} \frac{\partial}{\partial I} \frac{\partial \Phi_{2}}{\partial t}-\frac{\partial f}{\partial I} \frac{\partial}{\partial \Theta} \frac{\partial \Phi_{2}}{\partial t}=0 .
$$

Following the same approach in [27], $f$ can be expanded in terms of the small parameter $\beta=\frac{\tau_{B}}{\tau_{s}}$ to have

$$
f=f_{0}+\beta f_{1}+\beta^{2} f_{2}+\ldots
$$

where $f_{0}$ is the bounce average of $f$ over $\Theta$. Using expression (B.5), we substitute for $f$ in equation (B.4). To lowest order $(\mathcal{O}(1))$ in $\beta$, one finds

$$
\frac{\partial f_{0}}{\partial \Theta}=0
$$

To next order $(\mathcal{O}(\beta))$,

$$
\begin{aligned}
\frac{\partial f_{0}}{\partial t}+ & \beta \frac{\partial f_{1}}{\partial t}+\frac{\partial f_{0}}{\partial \Theta} \omega_{\text {Bounce }}+\beta \frac{\partial f_{1}}{\partial \Theta} \omega_{\text {Bounce }} \\
+ & \frac{\partial f_{0}}{\partial \Theta} \frac{\partial}{\partial I} \frac{\partial \Phi_{2}}{\partial t}+\beta \frac{\partial f_{1}}{\partial \Theta} \frac{\partial}{\partial I} \frac{\partial \Phi_{2}}{\partial t}-\frac{\partial f_{0}}{\partial I} \frac{\partial}{\partial \Theta} \frac{\partial \Phi_{2}}{\partial t} \\
& -\beta \frac{\partial f_{1}}{\partial I} \frac{\partial}{\partial \Theta} \frac{\partial \Phi_{2}}{\partial t}=0 .
\end{aligned}
$$

The second, sixth and eighth terms are on the order of $\beta^{2}\left(\mathcal{O}\left(\beta^{2}\right)\right)$ and can be neglected at this stage. Equation (B.6) shows that $f_{0}$ is independent of $\Theta$, which allows us to set the fifth term to zero. Therefore, we reach

$$
\frac{\partial f_{0}}{\partial t}+\beta \frac{\partial f_{1}}{\partial \Theta} \omega_{\text {Bounce }}-\frac{\partial f_{0}}{\partial I} \frac{\partial}{\partial \Theta} \frac{\partial \Phi_{2}}{\partial t}=0
$$

After averaging (B.8) over $\Theta$, the second and third terms vanish and we find

$$
\frac{\partial f_{0}}{\partial t}=0
$$

We define $f_{0}=\delta f+\left\langle F_{\text {eq }}\left(J_{\text {res }}(t)\right)\right\rangle$, where $<>$ denotes averaging over $\Theta$ and $f_{0}(t=0)=F_{\text {eq }}\left(J_{\text {res }}(t=0)\right)$. The uniformity assumption of the distribution function over the separatrix region assures $\left\langle F_{\text {eq }}\left(J_{\text {res }}(t)\right)\right\rangle=$ $F_{\text {eq }}\left(J_{\text {res }}(t)\right)$. Hence, $f_{0}(t)=\delta f+F_{\text {eq }}\left(J_{\text {res }}(t)\right)$. According to (B.9), $f_{0}$ should remain constant during frequency sweeping which gives

$$
\delta f=F_{\mathrm{eq}}\left(J_{\mathrm{res}}(t=0)\right)-F_{\mathrm{eq}}\left(J_{\mathrm{res}}(t)\right) .
$$

\section{Appendix C. Validation of the smallness of the perturbed potential energy change}

As illustrated in figure $\mathrm{C} 1$, we consider the case of a long range frequency chirping where the separatrix has approximately vanished. Therefore, we have $J_{\alpha,+}(t) \approx$ 0 . The change in the equilibrium energy $\left(E_{\mathrm{eq}, \alpha}\right)$ of the

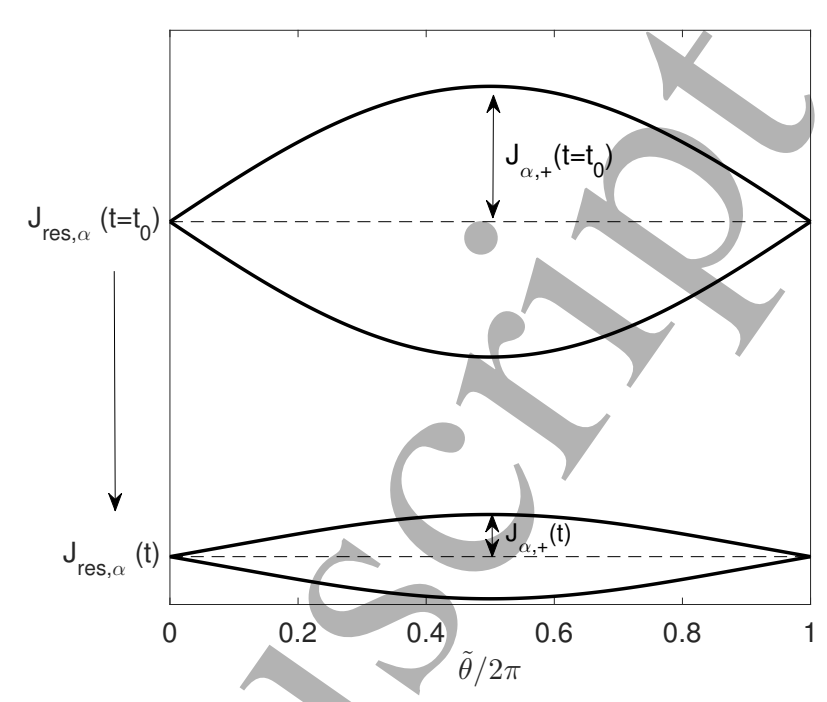

Figure C1. Schematic of a separatrix shrinkage with $J_{\alpha,+}(t) \approx$ 0 during long range frequency chirping.

trapped electrons in the BGK mode is

$$
\Delta E_{\mathrm{eq}, \alpha}=\frac{\partial H_{0, \alpha}}{\partial J_{\alpha}} \Delta J_{\mathrm{res}, \alpha}=\omega_{\mathrm{pe}} \Delta J_{\mathrm{res}, \alpha},
$$

where $\Delta J_{\text {res }, \alpha}=J_{\text {res }, \alpha}\left(t=t_{0}\right)-J_{\text {res }, \alpha}(t)$. Using equation (20), we find

$\Delta J_{\alpha}=J_{\alpha,+}\left(t=t_{0}\right)-J_{\alpha,+}(t) \approx \sqrt{\frac{A_{1,0} V_{\alpha, 1,1,0}}{\left|\Delta_{\alpha, 0}\right|}}$,

where, $t=t_{0}$ is denoted by the subscript 0 . The change in the perturbed energy ( $\left.E_{\text {perturbed, } \alpha}\right)$ of the electrons, which is the change in the perturbed potential energy, equals

$$
\Delta E_{\text {perturbed }, \alpha}=A_{1,0} V_{\alpha, 1,1,0} .
$$

We have claimed that if the change in $J_{\text {res, } \alpha}$ is greater than the change in the separatrix width, then $\Delta E_{\text {perturbed }, \alpha} \ll \Delta E_{\text {eq }, \alpha}$. Therefore, we have

$$
A_{1,0} V_{\alpha, 1,1,0} \ll \omega_{\text {pe }} \Delta J_{\text {res }, \alpha} .
$$

The above inequality can be written into

$$
\sqrt{\frac{A_{1,0} V_{\alpha, 1,1,0}}{\left|\Delta_{\alpha, 0}\right|}} \cdot \omega_{B} \ll \omega_{\mathrm{pe}} \Delta J_{\mathrm{res}, \alpha},
$$

where $\omega_{B}=\sqrt{A_{1,0} V_{\alpha, 1,1,0}\left|\Delta_{\alpha, 0}\right|}$ is the bounce frequency of trapped particles inside the separatrix in the BGK mode. Using equation (C.2) we find

$$
\frac{\Delta J_{\mathrm{res}, \alpha}}{\Delta J_{\alpha}} \gg \frac{\omega_{B}}{\omega_{\mathrm{pe}}} \approx \frac{\gamma_{l}}{\omega_{\mathrm{pe}}} .
$$

The RHS value is much less than unity $\left(\gamma_{l} \ll \omega_{\text {pe }}\right)$. Therefore, if the change in $J_{\text {res, } \alpha}$ is greater than the change in the width of the separatrix, the condition (C.6) is sufficiently satisfied. 